\title{
A Fibrational Method of Indexed Coinductive Data Types
}

\author{
Decheng MIAO, Chaoyang WANG, Xinsheng LIU, Yonglin LIANG
}

\begin{abstract}
As a fundamental issue in type theory, indexed coinductive data types (ICDT, for short) is of crucial importance, which is essentially semantic computing problem in programming. Based on fibrational method, this paper analyses semantic behaviours of ICDT and describes their universal coinductive rules. We executed some works in semantic computing and program logic of ICDT including their math structures and categorical properties. Example analyses prove the effectiveness of the proposed fibrational method and its applicability in program languages. Our work is based on fibration; a general math setting that can compute semantics automatically rather than depend on particular computing environments and syntactic forms of ICDT.
\end{abstract}

Keywords: category; coinductive data types; computation; fibration; programming

\section{INTRODUCTION}

The coinductive data type [1] analyses the semantic behaviours of data types in program languages and type theory; it is a dual concept of inductive data types with coalgebra as its math support [2, 3]. It observes the dynamic behaviours of languages during program execution. Inductive and coinductive data types form a complementary solution to improve the abilities of syntax construction and the semantic computation of program languages. ICDT, one kind of coinductive data type, has more semantic computing power and is capable of dealing with more complex data structures in programming. ICDT is an important part of programming and type theory. Traditional methods of ICDT, including category theory and coalgebra, make type theory models in the local Cartesian closed category, which then gives rise to two consequences: one is that indexed coinductive data types and the relation categories which describe their semantics co-exist in the same category together; another is that functor and its lifting are identical. Thus, this has some limitations to analyse semantic behaviours and depict coinductive rules.

Fibrations are a recently emerging trend in computer science, especially in categorical methods; they have many applications, such as database system modelling [4, 5], software specifications [6], and programming [7, 8]. In a fibrational setting, depicting the semantic behaviours of ICDT is no longer restricted to functions or morphisms, but is generalized to objects in a total category. More importantly, ICDTs and relation categories do not coexist in the same category any longer, but the latter constructs functor which is lifted in corresponding total categories to represent its abstracted semantic computing and program logic of ICDT. Hermida and Jacobs performed a great deal of work in this field [9].

We used fibrations to discuss ICDT in our works, firstly taking it to be the object set in the base category and taking its semantic behaviours to be the object set in total category; next we established the responsible relations in program logic directly between the ICDT and its semantic behaviours using an equation and quotient functor; lastly, we constructed the ICDT corecursive operations to describe abstract coinductive rules with universality using selffunctor constructed in base category and their corresponding lifting equation-preserving in the total category.

Our primary works have researched the semantic behaviours of ICDT and its coinductive rules using fibrations. The rest of the structure of our paper is as follows. In Section 2, we study some current related works. In Section 3 we introduce some basic concepts, such as the bifibration and reindexed functor. In Section 4, we present a single-sorted indexed fibration on slice categories to analyse the semantic behaviour of single-sorted ICDT and abstractly depict its coinductive rule with universality. In Section 5, we extend a discrete indexed object to the indexed category, developing single-sorted indexed fibration to many-sorted indexed fibration, and analysing the semantic behaviours of many-sorted ICDT and abstractly depicting its coinductive rule with universality. Lastly, we summarize our conclusions and discuss future researching work.

\section{RELATED WORKS}

As a coinductive data type whose abilities of semantic computation are stronger, ICDT takes coalgebra as its math foundation, introducing tools such as terminality and bisimulation to type theory, which has particular advantages in analyzing and describing the dynamic semantic behaviors of programming. From the perspective of document retrieval, Hagino maybe is the first one who worked on relationship between inductive and coinductive data type systematically using the dialgebras structure in [10]; his work laid the research foundation of the coinductive data type. However, there exist certain deficiencies in the polymorphism type system, the relationship between syntax construction and dynamic behaviors, the applications of coinduction data types.

Scholars' joint efforts provided the impetus for the further development of coinductive data types. Nogueira studied the relationship between inductive and coinductive data types and their application in polymorphism programming using bialgebra in [11]. Authors in [12] further melted the inductive and coinductive data type by $\lambda$ bialgebra and distributive laws, which explored the relationship between syntax construction and dynamic behaviors of data types. Poll et al. extended the works of Hagino based on sub-type and inheritance, and they obtained results on the connections of inductive and 
coinductive data types using the dual properties of algebra and coalgebra [13]. Greiner et al. brought coinductive principles in program languages. They studied coinductive data types in programming [1, 14]. Authors in [15] described indexed coinduction by co-recursion. All of the above results solved the aforementioned questions to some extent. Meanwhile, in the field of applications of coinductive data types, Gimenez studied some applications by Coq, the formal theory proof tool in [16]. Vene researched coinductive data types in the functional program language Haskell [17]. Most of the above results applied the methods of category theory and coalgebra, and those based on fibrations only focused on certain respects, such as the logic reason of coinductive data types and the validations of coinductive rules. For example, Hermida demonstrated the coinductive rules of final coalgebra with quotient types in [9]. Subsequently, Ghani et al. broke through the limitations of polynomial functors in [9], and developed their work to generic functors. Recently, they also presented $\lambda_{1}$ - fibration, constructed parameterized units of measure fibration UoM, and proved basic propositions of [18]. Based on [9], [19] proved the soundness of bisimulation coinduction in a fibrational setting, and provided a new categorical method of weak bisimulation by changing parameters. Chen and Urbatput forward a categorical method of automata theory, applied partial order set opfibration to study some concepts of the theory of algebraic automata in [20]. Based on seminal work by Worrell in [21], Hasuo et al. discussed coinductive predicates and final sequences in a fibration in [22], they identified some categorical 'size restriction' axioms that guarantee stabilization of final sequences after $\omega$ steps.

The current studies focus largely on coinductive data types; and the research on ICDT is still in its preliminary stage. There are many interesting questions in the field of semantics and programming; for instance, analyzing semantics behaviors and representing coinductive rules, particularly the latter are almost produced automatically. Those automatically produced coinductive rules lack a stable mathematical basis and concise formal specification. Our work focuses on semantics behaviors and coinductive rules of ICDT through fibrations. Comparing with conventional methods such as coalgebra and categoretical theory, this paper achieved the following: it analyzed semantic behaviors of ICDT succinctly using fibrations, it improved program languages processing and proving abilities for the semantic behaviors of ICDT, and it presented and described the coinductive rule with universality of ICDT. Meanwhile, all such works do not depend on specific computing enviroment, but they supply a sturdy mathematic basis as well as brief and unified description modes to semantics computing and logic of programming.

Using fibrational methods to study ICDT in formal languages extends and deepens the conventional ways of coinductive data types at categoretical theory, especially after coalgebras emerged, combining dually some category notions, including the fibration and the opposite fibration, reindexed functor and opposite reindexed functor, presents powerful vitality for fibrations in researching ICDT. There are wide development prospects in computer science theory foundations and engineering practice. Moreover, using fibrational method to research ICDT in formal semantics is not purely math research, but from the perspective of soft theory, integrating fibration method to the up-to-date results of type theory, formal languages and monads, this collaborates with fundamental study systematically and deeply for certain ICDT kernel problems including category interpreting of key notions, semantics behaviors and formal rules.

\section{FIBRATION AND OPFIBRATION \\ 3.1 Fibration and Reindexed Functor}

We assume readers have some categorical foundations, such as functor, adjunction, and natural transformation. Considering they have not presupposed the set theoretical models based on mathematical logic, some current basic math literature does not require all morphisms to be set when category is defined, but rather analyzing from the practical application perspective of computer science, we deem it is reasonable to confine all morphisms to a set. If all objects and morphisms can form two sets, respectively, in a category, the category is called a small category in [23]. The whole objects discussed in our works are in view of the concept of small category; readers can find other details for fibrations in [23-25]. For a category $\mathbb{C}$, let Obj $\mathbb{C}$ to be the objects set, Mor $\mathbb{C}$ to be the morph isms set.

Definition 1. Let $P: \mathbb{T} \rightarrow \mathbb{B}$ be a functor between two small categories $\mathbb{T}$ and $\mathbb{B}, f: C \rightarrow D \in$ Mor $\mathbb{B}$ and $P(Y)=D$. A morphism $u: X \rightarrow Y \in M$ or $\mathbb{T}$ is a Cartesian arrow of $f$ and $Y$, if the following three are satisfied: (1) $P(u)=f$. (2) For $\forall v: Z \rightarrow Y \in \boldsymbol{M o r} \mathbb{T}, \forall h: P(Z) \rightarrow C \in$ Mor $\mathbb{B}$, the diagram commutes, i.e. $f \cdot h=P(v)$.(3) There exists a unique $w: Z \rightarrow X \in M$ or $\mathbb{T}$ such that $u \cdot w=v$ and $P(w)=h$.

For the Cartesian arrow $u$ of $f$ and $Y$, we say $u$ lies above $f$; similarly, $Y$ lies above $D$. If $u$ is a cone [23] in category $\mathbb{T}$, then by the uniqueness of the cones morphism $w$, the Cartesian arrow $u$ in Definition 1 is also a universal cone i $\mathbb{T}$, namely, the limit cone. Accordingly, the vertex $X$ of universal cone $u$ is the terminal object of $u$ [26]. Then by the universal properties of universal cones, the Cartesianarrow $u$ is an isomorphism. We denote $f_{Y}^{\downarrow}$ for the Cartesian arrow $u$ of $f$ and $Y$ in Definition 1 for simplification.

Definition 2. Let $P: \mathbb{T} \rightarrow \mathbb{B}$ be a functor between two small categories $\mathbb{T}$ and $\mathbb{B}$. If there exists a Cartesian arrow $f_{Y}^{\downarrow}$ of $f$ and $Y$ for $\forall Y \in \boldsymbol{O} b j \mathbb{T}$ and $\forall f: C \rightarrow P(Y) \in$ Mor $\mathbb{B}$, then we call $P$ a fibration.

Using Definition 2, a fibration is a functor that ensures a large number of Cartesian arrows. For a fibration $P: \mathbb{T} \rightarrow \mathbb{B}, \mathbb{B}$ is called base category, $\mathbb{T}$ total category of $P$. For any object $C$ in $\boldsymbol{O} b j \mathbb{B}, \exists X \in \boldsymbol{O} b j \mathbb{T}$ and $k \in \boldsymbol{M}$ or $\mathbb{T}$, if satisfying $P(X)=C$ and $P(k)=i d_{c}$, then the sub-category $\mathbb{T}_{C}$ of $\mathbb{T}$ composed of $X$ and $k$ is called a fiber over $C$ [23], and $k$ is a vertical morphism. In fact, fiber $\mathbb{T}_{C}$ is a full subcategory of the total category $\mathbb{T}$.

Example 1. Let $\boldsymbol{S e t}$ be the set category, $\forall X \in \boldsymbol{O} b j \boldsymbol{S e t}$, a predicate over $X$ is a two-tuples $\langle X, P\rangle, P: X \rightarrow$ Set . For $\forall x \in X, P(x)$ forms a set, which describes the semantic behaviors of $x$, and $X$ is called the domain of predicate $<X$, 
$P>$. The predicates morphism from $\left\langle X, P>\right.$ to $\left\langle X^{\prime}, P^{\prime}>\right.$, is an ordered pair $\left(f, f^{\sim}\right):<X, P>\rightarrow<X^{\prime}, P^{\prime}>$, where $f: X \rightarrow X^{\prime}$ is a function in relevant predicate domain, and for $\forall x \in X, f^{\sim}: P(x) \rightarrow P^{\prime}(f(x)), P(x)$ is mapped to $P^{\prime}(f(\mathrm{x}))$. Predicates and their morphisms form the predicate category $\mathbb{P}$, and then predicate fibration Pre: $\mathbb{P} \rightarrow$ Set maps object $\langle X, P\rangle$ in total category $\mathbb{P}$ to $X$.

Let $g: X \rightarrow Y$ be a morphism in the base category $S e t$ on the predicate fibration Pre in Example 1, for $<Y, Q>\in \boldsymbol{O} b j \mathbb{P}$. We write $I d$ for the identify functor, then a Cartesian arrow $g_{<Y, Q\rangle}^{\downarrow}$ of $g$ and $\langle Y, Q>$ on the predicate category Pre is $\left(g, I d_{S e t}\right): Q g \rightarrow Q$.

Example 2. Write $\mathbb{B}^{\rightarrow}$ for the arrow category, domain functor dom: $\mathbb{B}^{\rightarrow} \rightarrow \mathbb{B}$ maps an object $f: X \rightarrow Y$ in $\mathbb{B}^{\rightarrow}$ to object $X$ in $\mathbb{B}$. We call dom a domain fibration above $\mathbb{B}$ . Functor cod: $\mathbb{B} \rightarrow \rightarrow \mathbb{B}$ maps an object $f: X \rightarrow Y$ in $\mathbb{B}^{\rightarrow}$ to the object $Y$ in $\mathbb{B}$. If $\mathbb{B}$ has pullbacks [23], then we call cod the codomain fibration.

For object $f: X \rightarrow Y$ in fiber $\mathbb{B}_{Y}$ on $Y$, we have morphism in the base category: $f^{\prime}: X^{\prime} \rightarrow Y \in$ Mor $\mathbb{B}$, so a Cartesian arrow of $f^{\prime}$ and $f$ on the codomain fibration cod is a pullback square of $f$ along $f$.

Example 3. Let category $\mathbb{B}$ have pullbacks, then $\operatorname{Sub}(\mathbb{B})$ is a category constituted by the sub-objects of $\mathbb{B}$; that is, objects of $\operatorname{Sub}(\mathbb{B})$ are mono-morphism equivalence classes. For $[f]: X>\rightarrow I \in \boldsymbol{O} b j \operatorname{Sub}(\mathbb{B})$ and another object $[g]: Y>\rightarrow J$, the morphism from $[f]$ to $[g]$ is $(I \rightarrow J):[f] \rightarrow[g] \in \operatorname{Mor} \operatorname{Sub}(\mathbb{B})$. We write $\alpha: I \rightarrow J, \beta: X \rightarrow Y$ , thus it satisfies diagram commuting, i.e., $\alpha \cdot[f]=[g] \cdot \beta$. Sub-object fibration $S: \operatorname{Sub}(\mathbb{B}) \rightarrow \mathbb{B}$, maps a monomorphism equivalence class $[f]$ to its codomain.

Write $f^{*}(Y)$ for domain of Cartesian arrow $f_{Y}^{\downarrow}$, then $f^{*}(Y)$ lies over $C$; that is, $Y \in \boldsymbol{O} b j \mathbb{T}_{D}, f^{*}(Y) \in \boldsymbol{O} b j \mathbb{T}_{C}$. Therefore we have the definition of a reindexed functor.

Definition 3. If a morphism $f: C \rightarrow D$ in the base category $\mathbb{B}$ is extended to be a functor $f^{*}: \mathbb{T}_{D} \rightarrow \mathbb{T}_{C}$ between fibers $\mathbb{T}_{D}$ and $\mathbb{T}_{C}$, then we call $f^{*}$ a reindexed functor induced by $f$.

Morphism $f$ is the relationship between ICDTs in the base category, and reindexed functor $f^{*}$ is a lifting of $f$ in the total category, which is related to their semantic behaviors.

\subsection{Opfibration and Opposite Reindexed Functor}

Definition 4. Let $P: \mathbb{T} \rightarrow \mathbb{B}$ be a functor between two small categories $\mathbb{T}$ and $\mathbb{B} ; f: C \rightarrow D \in$ Mor $\mathbb{B}$, $u: X \rightarrow Y \in M$ or $\mathbb{T}$. The morphism $u$ is called to be an opposite Cartesian arrow of $f$ and $X$ if three following conditions hold. (1) $P(u)=f$. (2) For $\forall v: X \rightarrow Z \in$ Mor $\mathbb{T}$ and $\forall h: D \rightarrow P(Z) \in M$ or $\mathbb{B}$, this satisfies diagram commuting, that is, $h \cdot f=P(v)$. (3) There exists a unique $w: Y \rightarrow Z \in M$ or $\mathbb{T}$ such that $w \cdot u=v$ and $P(w)=h$.
Similar to Definition 1 , if $u$ is a cocone [23] in category $\mathbb{T}$, then the opposite Cartesian arrow $u$ in Definition 4 is a universal cocone in $\mathbb{T}$ through the uniqueness of the cocones morphism $w$, namely, the colimit cocone. Accordingly, the vertex $Y$ of the universal cocone $u$ is the initial object of $u$ in [26], while the opposite Cartesian arrow $u$ is an isomorphism by the universal properties of universal cocones.

Definition 5. Let $P: \mathbb{T} \rightarrow \mathbb{B}$ be a functor between two small categories $\mathbb{T}$ and $\mathbb{B}$. If for $\forall X \in \boldsymbol{O} b j \mathbb{T}$ and $\forall f: P(X) \rightarrow D \in M$ or $\mathbb{B}$, there exists an opposite Cartesian arrow of $f$ and $X$, then we call $P$ an opfibration.

Definition 6. If the functor $P: \mathbb{T} \rightarrow \mathbb{B}$ between two small categories $\mathbb{T}$ and $\mathbb{B}$ is a fibration and an opfibration simultaneously, then it is a bifibration. Write $f_{\downarrow}^{X}$ for the opposite Cartesian arrow $u$ of $f$ and $X$ in Definition 4. Let ${ }^{*} f(X)$ be the codomain of $f_{\downarrow}^{X}$. Then we say ${ }^{*} f(X)$ lies above $D$, i.e., $X \in \boldsymbol{O} b j \mathbb{T}_{C},{ }^{*} f(X) \in \boldsymbol{O} b j \mathbb{T}_{D}$.

Definition 7. If a morphism $f: C \rightarrow D$ in the base category $\mathbb{B}$ is extended to be a functor ${ }^{*} f: \mathbb{T}_{C} \rightarrow \mathbb{T}_{D}$ between fibers $\mathbb{T}_{C}$ and $\mathbb{T}_{D}$, then ${ }^{*} f$ is an opposite reindexed functor induced by morphism $f$.

\subsection{Adjoint Properties of Reindexed and Opposite Reindexed Functor}

Definition 8. If $F \dashv G$ : $\mathbb{C} \rightarrow \mathbb{D}$ is a pair of adjoint functors, $\eta, \varepsilon$ is the unit and counit, respectively, and for $\forall X \in \boldsymbol{O}$ bj $\mathbb{C} \quad, \quad \forall Y \in \boldsymbol{O}$ bj $\mathbb{D} \quad, \quad \exists f: F(X) \rightarrow Y \in \boldsymbol{M}$ or $\mathbb{D}$, $\exists g: X \rightarrow G(Y) \in \operatorname{Mor} \mathbb{C}$, the transpose of $f$ and $g$ are $G(f) \eta_{X}$ and $\varepsilon_{Y} F(g)$, respectively.

Theorem 1. Let $P: \mathbb{T} \rightarrow \mathbb{B}$ be a fibration between the two small categories $\mathbb{T}$ and $\mathbb{B}$, then $P$ is a bifibration iff $\forall f: C \rightarrow D \in$ Mor $\mathbb{B}, f^{*}$ has a left adjoint functor ${ }^{*} f$.

Proof. $\Rightarrow$. Let ${ }^{*} f \dashv f^{*}: \mathbb{T}_{C} \rightarrow \mathbb{T}_{D}$ be a pair of adjoint functors, let $\eta$ be the unit, $\varepsilon$ be the counit, and $P: \mathbb{T} \rightarrow \mathbb{B}$ be a fibration between two small categories $\mathbb{T}$ and $\mathbb{B}$. Then $\exists Y \in \boldsymbol{O b j}_{D}$; we can construct a Cartesian arrow $f_{Y}^{\downarrow}: f^{*}(Y) \rightarrow Y$ whose codomain is $Y . \quad \exists X \in \boldsymbol{O} b j \mathbb{T}_{C}$, let $l: X \rightarrow^{*} f(X)$ be the morphism above $f$. In the following, we prove that $l$ is an opposite Cartesian arrow above $f$. This satisfies $l=f_{* f(X)}^{\downarrow} \cdot \eta_{X}$ by the adjoint property of ${ }^{*} \dashv f^{*}$, see Fig. 1. We write id for the identify morphism. If $g: X \rightarrow Y$ is another morphism above $f$, let $\phi: X \rightarrow f^{*}(Y)$ be the vertical morphism in $\mathbb{T}_{C}$, then we have $P(\phi)=i d_{C}$. By Definition 1, we have that $g=f_{Y}^{\downarrow} \cdot \phi$, where Cartesian arrow $f_{Y}^{\downarrow}$ is a universal cone, whose universal property ensures $\phi$ is the unique morphism from $g$ to $f_{Y}^{\downarrow}$. Let $\hat{\phi}$ be the transpose of $\phi$ under the adjunction ${ }^{*} f \dashv f^{*}$. Then $\hat{\phi}=\varepsilon_{Y} \cdot{ }^{*} f(\phi):{ }^{*} f(x) \rightarrow Y$, and $f^{*}(\hat{\phi}) \cdot \eta_{X}=\phi$. The universal 
property of universal cone $f_{Y}^{\downarrow}$ ensures the unique existence of $f^{*}(\phi)$; it satisfies diagram commuting, that is, $\hat{\phi} \cdot f_{*_{f(X)}}^{\downarrow}=f_{Y}^{\downarrow} \cdot f^{*}(\hat{\phi})$. Therefore, there exist equations $\hat{\phi} \cdot l=\hat{\phi} \cdot f_{*_{f}(X)}^{\downarrow} \cdot \eta_{X}=f_{Y}^{\downarrow} \cdot f^{*}(\hat{\phi}) \cdot \eta_{X}=f_{Y}^{\downarrow} \cdot \phi=g \quad$,i.e. $\quad g=\hat{\phi} \cdot l$.

Then the transpose $\hat{\phi}$ of $\phi$ is the unique morphism from $l$ to $g$, and $P(\hat{\phi})=i d_{D}$. Then by Definition $4, l$ is an opposite Cartesian arrow $f_{\downarrow}^{X}$ above $f$.

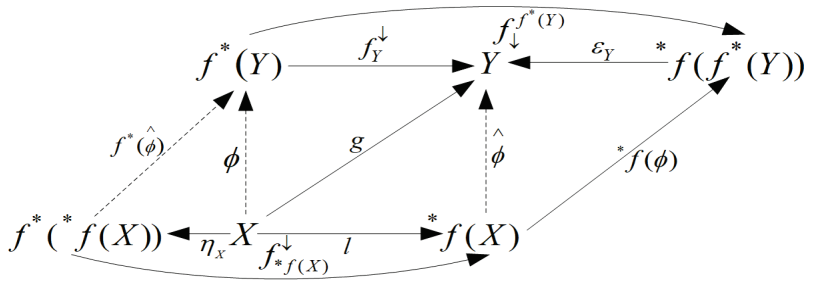

Figure 1 Proof of opposite Cartesian arrow

$\Leftarrow$. We assume $g: X \rightarrow Y \in \operatorname{Mor} \mathbb{T}$ lies above $f$, write $\mathbb{T}_{C}\left(X, f^{*}(Y)\right)$ for the set composed of morphisms above $C$ in fiber $\left.\mathbb{T}_{C}, T_{D}{ }^{*} f(X), Y\right)$ for the set composed of morphisms above $D$ in fiber $\mathbb{T}_{D}$. For $\forall k: X^{\prime} \rightarrow X \in \operatorname{Mor} \mathbb{T}_{C}$, $\forall h: Y \rightarrow Y^{\prime} \in \operatorname{Mor} \mathbb{T}_{D}$; because $P: \mathbb{T} \rightarrow \mathbb{B}$ is a bifibration, it has an one-to-one corresponding map $\varphi_{X, Y}: \mathbb{T}_{D}\left(^{*} f(X), Y\right) \rightarrow \mathbb{T}_{C}\left(X, f^{*}(Y)\right) . \quad$ We write $k^{o p}: X \rightarrow X^{\prime} \in \operatorname{Mor} \mathbb{T}_{C}$ for an opposite morphism of $k$. This satisfies that $\quad k^{o p} \cdot f_{\downarrow}^{X o p}=f_{\downarrow} X^{\prime} o p \cdot{ }^{*} f\left(k^{o p}\right) \quad$ and $i d_{f^{*}(Y)} \cdot f_{Y}^{\downarrow o p}=f_{Y}^{\downarrow o p} \cdot i d_{Y}$. Consequently, the left part of diagram in Fig. 2 commutes. Similarly, we have $i d_{X} \cdot f_{\downarrow}^{X o p}=f_{\downarrow}^{X o p} \cdot i d_{*_{f}(X)}$ and $f^{*}(h) \cdot f_{Y}^{\downarrow o p}=f_{Y^{\prime}}^{\downarrow o p}$, i.e., the right part of diagram in Fig. 2 also commutes. Hence $\varphi_{x, y}$ is a natural isomorphism. We thus prove ${ }^{*} f \dashv f^{*}$ by definition of adjoint functors in [26].

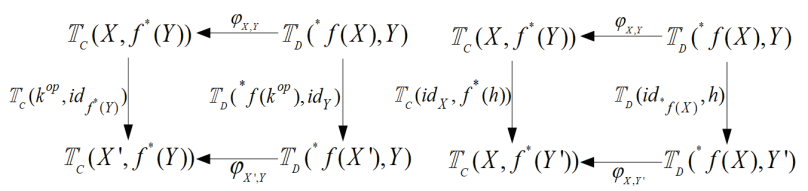

Figure 2 Proof of adjunction property

Remark 1. Theorem 1 gives a condition for determining if a functor is a bifibration. It also combines the adjoint property of the reindexed functor $f^{*}$ and the opposite reindexed functor ${ }^{*} f$ in the fibrational settings.

\section{SEMANTIC BEHAVIOURS OF SINGLE-SORTED ICDT AND ITS COINDUCTIVE RULE}

From the viewpoint of fibrations, single-sorted ICDT is an ICDT with discrete indexed objects, such as streams, lists, and trees. Based on the results from [27] and [28], this section constructs single-sorted indexed fibration by fibrations, analyzes semantic behaviors of single-sorted ICDT, and presents a coinductive rule of single-sorted ICDT with universality.

\subsection{Semantic Behaviours of Single-Sorted ICDT 4.1.1 Truth Functor and Relation Fibration}

Definition 9. Let $P: \mathbb{T} \rightarrow \mathbb{B}$ be a fibration between two small categories $\mathbb{T}$ and $\mathbb{B}$. For $\forall D \in \boldsymbol{O} b j \mathbb{B}$, if $\exists \mathbf{1}_{D} \in \boldsymbol{O} b j \mathbb{T}_{D}$ is a terminal object in fiber $\mathbb{T}_{D}$, and for $\forall f: C \rightarrow D \in$ Mor $\mathbb{B}$, $f^{*}\left(\mathbf{1}_{D}\right)$ is a terminal object in fiber $\mathbb{T}_{C}$, i.e., the reindexed functor $f^{*}$ preserves terminal objects, then we state that fibration $P$ has fibered terminal objects.

The fibered terminal object of predicate fibration Pre in Example 1 is a function map of all elements in the set $X$ to a singleton set. The fibered terminal object of the codomain fibration cod in Example 2 is an identity function. The sub-object fibration $S$ in Example 3 is an equivalence class of identity function.

Definition 10. Let $P: \mathbb{T} \rightarrow \mathbb{B}$ be a fibration between two small categories $\mathbb{T}$ and $\mathbb{B}$, and the functor $T_{P}: \mathbb{B} \rightarrow \mathbb{T}$ maps $\forall C \in \boldsymbol{O} b j \mathbb{B}$ to a terminal object in fiber $\mathbb{T}_{C}$. Then $T_{P}$ is a truth functor of fibration $P$. If $T_{P}$ has one right adjoint functor $\{-\}$, then we call $\{-\}$ a comprehension functor of $P$.

Let $\mathbf{1}_{\mathbb{B}}$ and $\mathbf{1}_{\mathbb{T}}$ be the terminal objects of the base category $\mathbb{B}$ and the total category $\mathbb{T}$, respectively. Then $P\left(\mathbf{1}_{\mathbb{T}}\right)=\mathbf{1}_{\mathbb{B}}$. For $\forall C \in \boldsymbol{O} b j \mathbb{B}$, there exists a unique morphism $u: C \rightarrow \mathbf{1}_{\mathbb{B}}$ such that $T_{P}(C) \cong u^{*}\left(\mathbf{1}_{\mathbb{T}}\right)$. For $\forall f: C \rightarrow D \in$ Mor $\mathbb{B}$, we have $f^{*}\left(T_{P}(D)\right) \cong T_{P}(C)$, and the truth functor $T_{P}$ maps $f$ to its Cartesian arrow $f_{T_{P}(D)}^{\downarrow}$ in total category $\mathbb{T}$.

Definition 11. Let $P: \mathbb{T} \rightarrow \mathbb{B}$ be a fibration between two small categories $\mathbb{T}$ and $\mathbb{B}$; its base category $\mathbb{B}$ has products. Let $\Delta: \mathbb{B} \rightarrow \mathbb{B}$ be a diagonal endo-functor above $\mathbb{B}$, which maps $\forall C \in O b j \mathbb{B}$ to the product object $C \times C$. Then the pullback of $P$ along $\Delta$ forms fibration $\operatorname{Rel}(P): \operatorname{Rel}(\mathbb{T}) \rightarrow \mathbb{B}, \operatorname{Rel}(P)$ is called to be a relation fibration of $P$.

The object of the total category $\operatorname{Rel}(\mathbb{T})$ on $\operatorname{Rel}(P)$ is relation $(C, D)$; for another object $\left(C^{\prime}, D^{\prime}\right)$, let $f: C \rightarrow C^{\prime}$ and $g: D \rightarrow D^{\prime}$ be two morphisms. Then $(f, g):(C, D) \rightarrow\left(C^{\prime}, D^{\prime}\right) \in \operatorname{Mor} \operatorname{Rel}(\mathbb{T})$. The relation fibration $\operatorname{Rel}(P)$ in Fig. 3 maps the relation $(C, D)$ to object $C$ in the base category $\mathbb{B}$; functor $\Pi$ maps $(C, D)$ to object $D$ in $\mathbb{T}$ , and $P(D)=\Delta(C)$. Moreover, the property pullbackpreserving of Definition 11 ensures that fiber $\operatorname{Rel}(\mathbb{T})_{C}$ above $C$ on $\operatorname{Rel}(P)$ is an isomorphism to fiber $\mathbb{T}_{C \times C}$ above $C \times C$ on $P$, i.e., $\operatorname{Rel}(\mathbb{T})_{C} \cong \mathbb{T}_{C \times C}$.

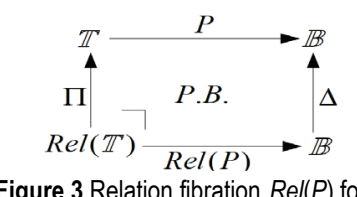


The procedure of building new fibration using specific fibration is called to be change of base. For instance, we can build $\operatorname{Rel}(P)$ using the change of base from $P$ in Definition 11. The change of base keeps construction including preserving fibered terminal objects in [26]; if the fibration $P$ has one truth functor $T_{P}$, then its relation fibration $\operatorname{Rel}(P)$ has truth functor $T_{\operatorname{Rel}(P)}$. Meanwhile, we can gain $T_{\operatorname{Rel}(P)}(\mathrm{C})=T_{P}(C \times C)$. The predicate fibration Pre in Example 1 constructs a relation fibration $\operatorname{Rel}($ Pre $)$ using a change of base, and its truth functor maps set $X$ to a twotuple relation $R: X \times X \rightarrow$ Set, i.e., it maps each ordered pair $\left(x, x^{\prime}\right)$ to a singleton set $\{*\}$.

Theorem 2. Let $P: \mathbb{T} \rightarrow \mathbb{B}$ be a bifibration between two small categories $\mathbb{T}$ and $\mathbb{B}$; base category $\mathbb{B}$ has pullbacks. If for each pullback square in $\mathbb{B}$, natural transformation ${ }^{*} s \cdot t^{*} \rightarrow g^{*} \cdot{ }^{*}$ is an isomorphism, then $P$ satisfies Beck-Chevalley condition.

Proof. Let $\eta_{f}$ be the unit of the adjoint functor ${ }^{*}{ }_{f} \dashv f^{*}$ , and let $\varepsilon_{s}$ be the counit of the adjoint functor ${ }^{*} s \dashv s^{*}$ (Fig. 4). Then $\eta_{f}=I d_{\mathbb{T}_{B}}, \varepsilon_{s}=I d_{\mathbb{T}_{C}}$. The following equation holds: $\left.{ }^{*} s \cdot t^{*}\right) \cdot \eta_{f}=\left({ }^{*} s \cdot t^{*}\right) \cdot\left(f^{*} \cdot{ }^{*} f\right)$, and the pullback square in Fig. 4 satisfies diagram commuting: $f \cdot t=g \cdot s$, and $s$ is a pullback of $f$ along $g, t$ is a pullback of $g$ along $f$. Using the pullback property of the reindexed functor, we have $t^{*} \cdot f^{*} \cong s^{*} \cdot g^{*} \quad$. So $\left({ }^{*} s \cdot t^{*}\right) \cdot\left(f^{*} \cdot{ }^{*} f\right)={ }^{*} s \cdot\left(t^{*} \cdot f^{*}\right) \cdot{ }^{*} f \cong{ }^{*} s \cdot\left(s^{*} \cdot g^{*}\right) \cdot{ }^{*} f \quad, \quad$ and ${ }^{*} s \cdot\left(s^{*} \cdot g^{*}\right) \cdot{ }^{*} f=\left({ }^{*} s \cdot s^{*}\right) \cdot\left(g^{*} \cdot{ }^{*} f\right)=\varepsilon_{s} \cdot\left(g^{*} \cdot{ }^{*} f\right)=\left(g^{*} \cdot{ }^{*} f\right)$, that is, ${ }^{*} s \cdot t^{*} \cong g^{*} \cdot{ }^{*} f$. Hence the natural transformation ${ }^{*} s \cdot t^{*} \rightarrow g^{*} \cdot{ }^{*} f$ is an isomorphism.

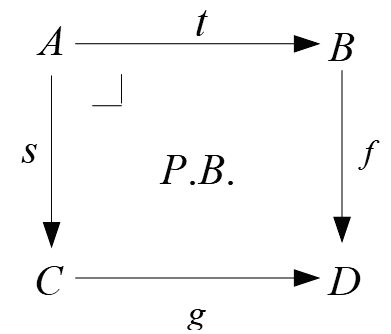

Figure $4 \mathrm{~A}$ pullback square in base category $\mathbb{B}$

Remark 2. In other word, based on the pullback square in the base category on a bifibration, Theorem 2 defines a natural transformation whose functors preserve the structure between corresponding fibers in the total category $\mathbb{T}$. The theorem further ensures the reindexed functor and the opposite reindexed functor satisfy appropriate properties of diagram commuting. For example, the predicate fibration Pre in Example 1 and the codomain fibration cod in Example 2 both satisfy the Beck-Chevalley condition in Theorem 2.

Definition 12. Let $P: \mathbb{T} \rightarrow \mathbb{B}$ be a bifibration which is satisfying the Beck-Chevalley condition; the base category $\mathbb{B}$ has products, furthermore, $T_{P}$ is one truth functor of $P$. For any $\forall C \in \boldsymbol{O} b j \mathbb{B}$ the active function $\delta_{C}: C \rightarrow C \times C$ of natural transformations $\delta: I d_{\mathbb{B}} \rightarrow \Delta$ on $C$ extend one opposite reindexed functor ${ }^{*} \delta$. Meanwhile,
$E q_{P}: \mathbb{B} \rightarrow \operatorname{Rel}(\mathbb{T})$ is one equation functor of $P$, we can obtain $E q_{P}={ }^{*} \delta \cdot T_{P}$.

The truth functor $T_{P}$ of $T$ is mapping $C$ to one terminate object $T_{P}(C)$ in fiber $\mathbb{T}_{C}$. From Definition 11 , we can obtain that $\operatorname{Rel}(P)$ is the change of base of $P$ along $\Delta$. So if fibration $P$ has one fibered terminate object, then its relation fibration $\operatorname{Rel}(P)$ has one fibered terminate object too. If the opposite reindexed functor ${ }^{*} \delta$ can map terminate object $T_{P}(C)$ to the ${ }^{*} \delta\left(T_{P}(C)\right)$, then ${ }^{*} \delta\left(T_{P}(C)\right) \in \boldsymbol{O b j}\left(\mathbb{T}_{C \times C} \cong \operatorname{Rel}\left(\mathbb{T}^{\prime}\right)_{C}\right)$, and the equation functor $E q_{P}$ of $P$ also map $\forall f \in$ Mor $\mathbb{B}$ to only one unique morphism on $f \times f$ which is determined by $\delta_{f}$ and $\left(\delta_{C}\right)_{\downarrow}^{T_{P}(C)}$. The intuitional implication of the equation functors is that identical parameters have identical results [9]. Take the predicate fibration Pre in Example 1 as an example; the object in its fiber $\operatorname{Rel}(\mathbb{T})_{C}$ is the equation relation $R: X \times X \rightarrow$ Set , and $E q_{P r e}(C)\left(x, x^{\prime}\right)=1$ if $x \neq x^{\prime}$; and $E q_{P r e}(C)\left(x, x^{\prime}\right)=0$ if otherwise.

\subsubsection{Single-Sorted Indexed Fibration and its Equation Functor}

Theorem 3. Let $P: \mathbb{T} \rightarrow \mathbb{B}$ be a fibration or bifibration between two small categories $\mathbb{T}$ and $\mathbb{B}$. Then $T_{P}: \mathbb{B} \rightarrow \mathbb{T}$ is one truth functor for $P$. So, $\exists I \in \boldsymbol{O} b j \mathbb{B}, I$ is one discrete indexed target on the base category $\mathbb{B}$. Assume the singlesorted indexed functor $P / I, \mathbb{T} / T_{P}(I) \rightarrow \mathbb{B} / I$ tobe $P / I(u)=P(u): P(Y) \rightarrow I \in \boldsymbol{O} b j \mathbb{B} / I$, for $\forall u: Y \rightarrow T_{P}(I) \in \boldsymbol{O} b j \mathbb{T} / T_{P}(I)$. Then the single-sorted indexed functor $P / I$ is also a fibration or bifibration.

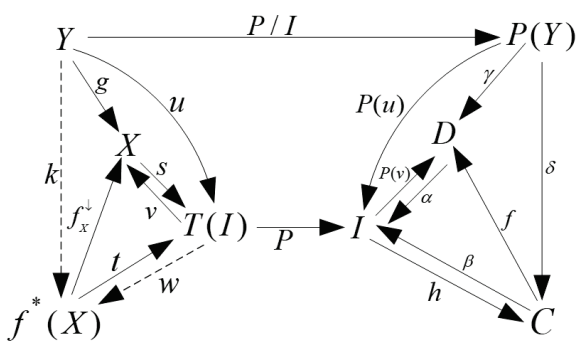

Figure 5 Cartesian morphism $f_{X}^{-}$of $P$ / / above $f$

Proof. For $\forall f: C \rightarrow D \in$ Mor $\mathbb{B}$, we can get one Cartesian arrow $f_{X}^{\downarrow}: f^{*}(X) \rightarrow X$ on $f$ about fibration $P$, which satisfies $P(X)=D$. We also obtain an only morphism $w: T_{P}(I) \rightarrow f^{*}(X)$ so have $v=f_{X}^{\downarrow} \cdot w$ and $P(v)=f \cdot h$ (Fig. 5). On the supposition that we have $\alpha: D \rightarrow I \in \boldsymbol{O} b j \mathbb{B} / I$, $\beta: C \rightarrow I \in \boldsymbol{O} b j \mathbb{B} / I$. So $\gamma: P(u) \rightarrow \alpha=P(Y) \rightarrow D \in$ Mor $\mathbb{B} / I$, $\delta: P(u) \rightarrow \beta=P(Y) \rightarrow C \in M$ or $\mathbb{B} / I$, and the diagram commutes: $\gamma=f \cdot \delta$. In the total category $\mathbb{T} / T_{P}(I)$ on functor $P / I$, $s: X \rightarrow T_{P}(I) \in \boldsymbol{O} b j \mathbb{T} / T_{P}(I), t: f^{*}(X) \rightarrow T_{P}(I) \in \boldsymbol{O} b j \mathbb{T} / T_{P}(I)$, we have $g: u \rightarrow s=Y \rightarrow X \in \operatorname{Mor} \mathbb{T} / T_{P}(I)$. So we can obtain an only morphism, such that the diagram commutes, i.e. 
$g=f_{x}^{\downarrow} \cdot k$. By Definition $1 f_{x}^{\downarrow}$ is a Cartesian arrow of $f$ on functor $P / I$. So if $P$ is a fibration, then the single-sorted functor $P / I$ is also a fibration.

Let $m: Z \rightarrow T_{P}(I) \in \boldsymbol{O} b j \mathbb{T} / T_{P}(I)$. So $P / I(m)=\alpha$ using functor $P / I$. Meanwhile, assume $f_{\downarrow}^{Z}: Z \rightarrow{ }^{*} f(Z)$ one oppositive cartesian arrow for $f$ about $P$ (Fig. 6). The commuting diagrams in the slice categories $\mathbb{B} / I$, $\alpha=\beta \cdot f$, and we can obtain one only morphism $n:{ }^{*} f(Z) \rightarrow T_{P}(I)$ in the total categories $\mathbb{T} / T_{P}(I)$ over functor $P$ / I such that the diagrams commute, $m=n \cdot f_{\downarrow}^{Z}$. By Definition $4, f_{\downarrow}^{Z}$ is an opposite Cartesian arrow of $f$ on functor $P / I$. Namely, if $P$ is an opposite fibration, then the single-sorted indexed functor $P / I$ is also an opposite fibration.

Therefore, if $P$ is a fibration or bifibration, then the single-sorted indexed functor $P / I$ is also a fibration or bifibration

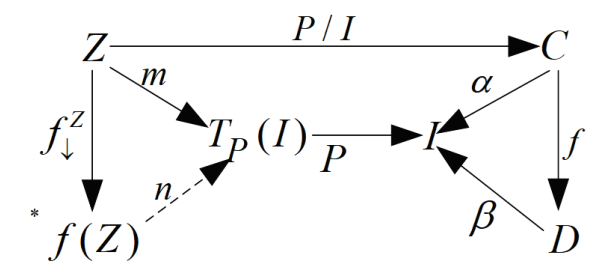

Figure 6 Opposite Cartesian morphism $f_{\downarrow}^{Z}$ of $P$ / I above $f$

Remark 3. Theorem 3 proves that the single-sorted indexed fibration $P / I$ and fibration $P$ have the same properties of fibration or bifibration; we also provide the definition of a single-sorted indexed fibration. In fact, a change of base of $P$ along the domain functor dom $: \mathbb{B} / I \rightarrow \mathbb{B}$ in Example 2 can construct a single-sorted indexed fibration $P / I: \mathbb{T} / T_{P}(I) \rightarrow \mathbb{B} / I$. For $\forall \alpha: C \rightarrow I \in \boldsymbol{O} b j \mathbb{B} / I$, the fiber $\mathbb{T}_{C}$ above $C$ on $P$ is an isomorphism to the fiber $(\mathbb{T} / T(I))_{\alpha}$ above $\alpha$ on $P / I$ [28], and if $P$ has a truth functor, then the single-sorted indexed fibration $P / I$ constructed by $P$ also has a truth functor.

Any $\forall \alpha: C \rightarrow I \in \boldsymbol{O} b j \mathbb{B} / I$, we presume two pull-backs of $\alpha$ along $\alpha$ to be $i$ and $j$, separately. So the productive object of $\alpha \times \alpha$ is $\alpha \cdot i$ or $\alpha \cdot j$. Namely, the productive object $\mathbb{B} / I$ in those slice categories is ascertained by the pullback. Analogously, for definition 11, the subsequent result is the concept of a relation fibration of the singlesorted indexed fibration $P / I$.

Definition 13. Assume $P / I: \mathbb{T} / T_{P}(I) \rightarrow \mathbb{B} / I$ to be a single-sorted indexed fibration. The base categories $\mathbb{B} / I$ have product. Presume $\Delta / I: \mathbb{B} / I \rightarrow \mathbb{B} / I$ to be one bidiagonal selffunctor in the sliced category $\mathbb{B} / I$. So $\Delta / I$ mapping $\forall \alpha \in \mathbb{B} / I$ to the product object $\alpha \times \alpha$. Then pullbacks of $P / I$ along $\Delta / I$ makes one fibration $\operatorname{Rel}(P / I): \operatorname{Rel}\left(\mathbb{T} / T_{P}(I)\right) \rightarrow \mathbb{B} / I$. At the same time, $\operatorname{Rel}(\mathrm{P} /$ $I)$ is one relation fibration for $P / I$.

For an object $R \in \operatorname{Obj} \operatorname{Rel}\left(\mathbb{T} / T_{P}(I)\right)$ above $\alpha$ on $\operatorname{Rel}(\mathrm{P} /$ $I)$, an object $R^{\prime} \in \boldsymbol{O b j} \mathbb{T} / T_{P}(I)$ above $\alpha \times \alpha$ on $P / I$ and an object $R^{\prime \prime} \in \boldsymbol{O b j T}$ above $\operatorname{dom}(\alpha \times \alpha)$ on $P$, there exists the isomorphism $R \cong R^{\prime} \cong R^{\prime \prime}$ in [28]. The action function of $\alpha$ on the natural transformation $\delta / I: I d_{\mathbb{B} / I} \rightarrow \Delta / I$ is $(\delta / I)_{\alpha}: C \rightarrow \operatorname{dom}(\alpha \times \alpha)$. Then the intuitional meaning of the natural transformation $\delta / I$ is a morphism from one object to another object in the slice category $\mathbb{B} / I$. Similarly, for Definition 12, the following defines the equation functor of a single-sorted indexed fibration $P / I$.

Definition 14. Let $P: \mathbb{T} \rightarrow \mathbb{B}$ be a bifibration satisfying Beck-Chevalley condition between two small categories $\mathbb{T}$ and $\mathbb{B}$, where $P$ has the truth functor, and base category $\mathbb{B}$ has the product. Let the truth functor of a single-sorted index fibrations $P / I$ be $T_{P / I}$. So $E q_{P / I}={ }^{*}(\delta / I) \cdot T_{P / I}: \mathbb{B} / I \rightarrow \operatorname{Rel}\left(\mathbb{T} / T_{P}(I)\right)$ is called to be one equation functor for $P / I$.

The equation functor $E q_{P / I}$ maps the object $\alpha: C \rightarrow I$ in the slice category $\mathbb{B} / I$ to a unique morphism ${ }^{*}(\delta / I)_{\alpha} \cdot T_{P / I}(C) \rightarrow T_{P}(I)$ above $\alpha \times \alpha$. The following constructs the quotient functor using the single-sorted indexed fibration $P / I$.

\subsubsection{Quotient Functor and its Lifting}

Let truth functor $T_{P}: \mathbb{B} \rightarrow \mathbb{T}$ of fibration $P: \mathbb{T} \rightarrow \mathbb{B}$ be substituted with the equation functor $E q_{P}: \mathbb{B} \rightarrow \operatorname{Rel}(\mathbb{T})$ of $P$. $P$ is displaced by its relation fibration $\operatorname{Rel}(P)$. Next, applying Theorem 3, we make another fibration, i.e., $\operatorname{Rel}(P) / I: \operatorname{Rel}(\mathbb{T}) / E q_{P}(I) \rightarrow \mathbb{B} / I$, any $\forall R \in \operatorname{ObjRel}(\mathbb{T})$. Here, $\operatorname{Rel}(P) / I$ can map $\alpha: R \rightarrow E q_{P}(I)$ to be $\alpha^{\prime}: Q R \rightarrow I$, and $\alpha^{\prime}$ is one transpose of $\alpha$ to the adjoint functors $Q \dashv E q_{P}$.

Definition 15. Assume adjoint functors $\tau \dashv$ $\sigma: \operatorname{Rel}\left(\mathbb{T} / T_{P}(I)\right) \rightarrow \operatorname{Rel}(\mathbb{T}) / E q_{P}(I) \quad$ satisfy commuted diagrams, namely, $\operatorname{Rel}(P / I)=\operatorname{Rel}(P) / I \cdot \tau, \quad$ and $\operatorname{Rel}(P) / I=\operatorname{Rel}(P / I) \cdot \sigma$. Meanwhile, $\operatorname{Rel}(P) / I$ have the right adjoint functors $E q_{(P / I)}$ such that $E q_{(P / I)}=\tau \cdot E q_{P / I}$.Then $\operatorname{Rel}(P) / I \cdot \tau \dashv \sigma \cdot E q_{(P / I)}$. And $\operatorname{Rel}(P / I) \cdot \sigma$ is called to be the quotient functor of the single-sorted indexed fibration $P / I$, We write $\operatorname{Rel}(P) / I \cdot \tau$ to $Q_{P / I}$. And we have $Q_{P / I} \dashv E q_{P / I}$.

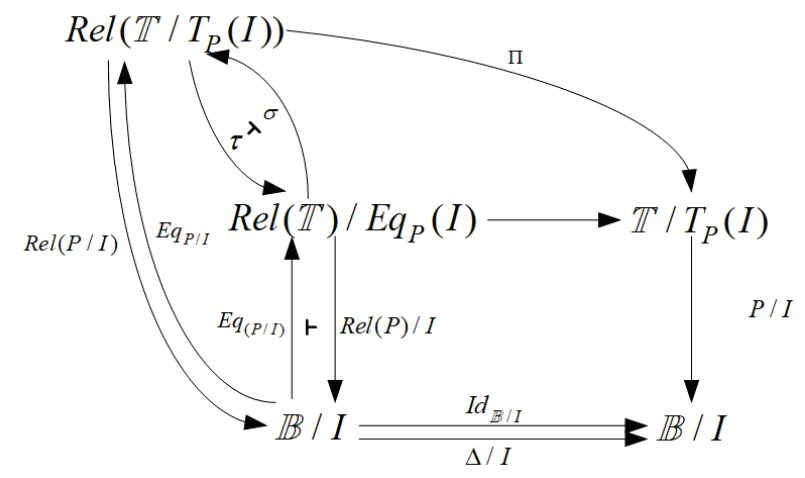

Figure 7 Construction of quotient functor $Q_{p / l}$

Let $\quad \forall R=(C, D) \in \operatorname{Obj} \operatorname{Rel}\left(\mathbb{T} / T_{P}(I)\right) \quad$. Then $Q_{P / I}(C, D)=C$ (Fig. 7). We have $\Pi(C, D)=D$, for an object 
$f: D \rightarrow T_{P}(I) \in \boldsymbol{O} b j \mathbb{T} / T_{P}(I)$; we have $P / I(f)=P(D) \rightarrow I$, and there exists an object $g: C \rightarrow I \in \boldsymbol{O} b j \mathbb{B} / I$, $\Delta / I(g)=g \times g$; therefore: $\operatorname{dom}(g \times g)=P(D)$.

Definition 16. Let $P: \mathbb{T} \rightarrow \mathbb{B}$ be one bi-fibration satisfies Beck-Chevalley requirement with a truth functor $T_{P}$ among small category $\mathbb{T}$ and $\mathbb{B}$. Base categories $\mathbb{B}$ have product and pullback. The functor $P / I: \mathbb{T} / T_{P}(I) \rightarrow \mathbb{B} / I$ is a single-sorted index fibration for $P$. We make a relational fibration $\operatorname{Rel}(P / I)$, an equational functors $E q_{P / I}$ with a quotient functor $Q_{P / I}$ of $P / I$. Presume $F$ to be one self functor into the base categories $\mathbb{B} / I$ over $\operatorname{Rel}(P / I)$. Then $F^{\perp}$ is a self functor into the total categories $\operatorname{Rel}\left(\mathbb{T} / T_{P}(I)\right)$ over $\operatorname{Rel}(P / I)$. In case $F^{\perp}$ is satisfying commuted diagrams, i.e., $\operatorname{Rel}(P / I) \cdot F^{\perp}=F \cdot \operatorname{Rel}(P / I)$, some isomorphism expresses satisfy, $E q_{P / I} \cdot F \cong F^{\perp} \cdot E q_{P / I}$ and $F \cdot Q_{P / I} \cong Q_{P / I} \cdot F^{\perp}$.Then we call $F^{\perp}$ one lifting which is equation-preserving for $F$ on $\operatorname{Rel}(P / I)$ into total category $\operatorname{Rel}\left(\mathbb{T} / T_{P}(I)\right)$

\subsubsection{Semantic Behaviours of Single-Sorted ICDT}

Any $\forall \alpha: C \rightarrow I \in \boldsymbol{O} b j \mathbb{B} / I \quad, \quad$ one $F$ coalgebras $(\alpha, r: \alpha \rightarrow F(\alpha))$ is made using action of selffunctor $F$. We call $\alpha$ the carrier of $F$ coalgebras, the morphism between $(\alpha, r)$ and another $F$ - coalgebra $(\beta, t: \beta \rightarrow F(\beta))$ is the morphism $f: \alpha \rightarrow \beta$ between their carriers, which satisfies the diagram commutes; that is, $t \cdot f=F(f) \cdot r . F$ coalgebras category is composed of $F$ - coalgebras and corresponding morphisms, writes Colag $_{F}$. If the terminal $F$ - coalgebra ( $v F$, out: $v F \rightarrow F(v F)$ ) exists, it is up to a unique isomorphism with the universal properties, which are determined by terminal coalgebra. The universal properties are our primary tool to research the semantic behaviors and coinductive rules of ICDT.

The single-sorted ICDT $v F$, which is also the carrier of final $F$ - coalgebras, is the max fixed points of the functor $F$. The functor $F$ is the syntax destructors of $v F$. The corresponding morphism out describes a type of semantic behavior of $v F$ during the syntax destruction externally. We apply equation functors $E q_{P / I}$ of the single-sorted indexed fibration $P / I$, it mapped $F$ - coalgebra $(\alpha, r)$ to an $F^{\perp}$ - coalgebra,

$E q_{P / I}(\alpha, r)=\left(E q_{P / I}(\alpha), E q_{P / I}(r): E q_{P / I}(\alpha) \rightarrow E q_{P / I}(F(\alpha))\right.$

$\left.\cong F^{\perp}\left(E q_{P / I}(\alpha)\right)\right)$. Accordingly, $E q_{P / I}(v F)$ is the carrier of terminal $F^{\perp}$ - coalgebra. Therefore, the equation functor $E q_{P / I}$ preserves terminal objects.

Write $\operatorname{Coalg}\left(E q_{P / I}\right)$ for the functor from $\operatorname{Coalg}_{F}$ to Coalg $_{F^{\perp}}$,which maps all objects and their morphisms in the base category $\mathbb{B} / I$ over relation fibration $\operatorname{Rel}(P / I)$ to ones into the total categories $\operatorname{Rel}\left(\mathbb{T} / T_{P}(I)\right)$ using the equation functor $E q_{P / I}$. Then the functor $\operatorname{Coalg}\left(E q_{P / I}\right)$ establishes a relationship between Coalg $_{F}$ and Coalg $_{F^{\perp}}$.
If $\left(E q_{P / I}(v F), o u t^{\perp}: E q_{P / I}(v F) \rightarrow F^{\perp}\left(E q_{P / I}(v F)\right)\right)$ is a final $F^{\perp}$ - coalgebras into the total categories $\operatorname{Rel}\left(\mathbb{T} / T_{P}(I)\right)$ over relation fibration $\operatorname{Rel}(P / I)$, then the out $t^{\perp}$ is one homo-morphism image for out with the act of the corresponding functor $\operatorname{Coalg}\left(E q_{P / I}\right)$, namely, we have $\operatorname{Coalg}\left(E q_{P / I}\right)($ out $)=o u t^{\perp}$. The final properties of final $F^{\perp}$ coalgebras ensure $o u t^{\perp}$ determines an only isomorphism, providing convenience in analyzing semantic behaviors accurately and exactly depicting the coinductive rule of single-sorted ICDT.

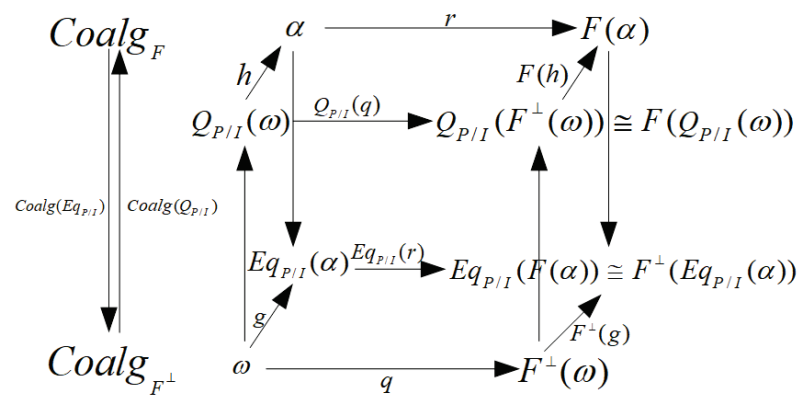

Figure 8 Adjoint properties of $\operatorname{Coalg}\left(E_{P / I}\right)$ and $\operatorname{Coalg}\left(Q_{P \prime \prime}\right)$

Similarly, write $\operatorname{Coalg}\left(Q_{P / I}\right)$ for the functor from Coalg $_{F^{\perp}}$ to $\operatorname{Coalg}_{F}$. Then we have $\operatorname{Coalg}\left(Q_{P / I}\right)-1$ $\operatorname{Coalg}\left(E q_{P / I}\right)$ by the adjoint property of the adjoint functor [9]. For each $F^{\perp} \quad$ - coalgebra $\left(\omega, q: \omega \rightarrow F^{\perp}(\omega)\right), \omega: X \rightarrow T_{P}(I) \in \operatorname{ObjRel}\left(\mathbb{T} / T_{P}(I)\right)$ $\operatorname{Coalg}\left(Q_{P / I}\right)(q)=Q_{P / I}(\omega) \rightarrow Q_{P / I}\left(F^{\perp}(\omega)\right) \cong F\left(Q_{P / I}(\omega)\right)$, that is, $\operatorname{Coalg}\left(Q_{P / I}\right)(q)=Q_{P / I}(q)$. So $Q_{P / I}(q)$ be one of homomorphism images of $q$ with the act of the functor $\operatorname{Coalg}\left(Q_{P / I}\right)$ (Fig. 8). If the morphism $g: \omega \rightarrow E q_{P / I}(\alpha)$ is a $F^{\perp}$ - coalgebras morphism from $q$ to $E q_{P / I}(r)$, then the $F$ coalgebras morphism $h: Q_{P / I}(\omega) \rightarrow \alpha$ from $Q_{P / I}(q)$, to $r$ is an $F$ - coalgebras homomorphisms about $g$. Similarly, $g$ is an $F^{\perp}$ - coalgebra homomorphism above $h$.

The left adjoint $\operatorname{Coalg}\left(Q_{P / I}\right)$ of functors $\operatorname{Coalg}\left(E q_{P / I}\right)$ causes a presentative mutual deduction relations among $F$ - coalgebras whose carrier is $Q_{P / I}(\omega), F^{\perp}$ - coalgebras whose carriers are $\omega$, providing a concise and uniform model ways to the math specification of the coinductive rule of a single-sorted ICDT. The single-sorted ICDT $v F$ is the carrier of terminal coalgebra, if functor $\operatorname{Coalg}\left(E q_{P / I}\right)$ preserves terminal objects, then the lifting equationpreserving $F^{\perp}$ of $F$ on $\operatorname{Rel}(P / I)$ generates a sound coinductive rule.

\subsection{Coinductive Rule of Single-Sorted ICDT}

One fibration equipping a quotient functor and equation functor, the math specifiction of coinductive rule is coherent to its semantics behaviors analysis of ICDT on this fibration [9]. If $P: \mathbb{T} \rightarrow \mathbb{B}$ and $P / I: \mathbb{T} / T_{P}(I) \rightarrow \mathbb{B} / I$ content the conditions of Definition 16, and let $F$ be a self functor into the base category $\mathbb{B} / I$ over the relation fibration $\operatorname{Rel}(P / I)$ for $P / I$, let $v F$ be one carrier of final $F$ 
- coalgebras, $F$ have its lifting which is equation-preserving $F^{\perp}$, then $P / I$ have the coinductive rules whose carrier is the single-sorted ICDT $v F$. This provides a sound basis of validity judgment for $F^{\perp}$ applying $F$ - coalgebra to generate a coinductive rule on a single-sorted ICDT. That is, if the single-sorted index fibration $P / I$ equipping quotient functor and equation functor depicts semantics behaviors of the single-sorted ICDTs, their coinductive rules on account of final $F$ - coalgebras are available in the procedure of semantics behaviors descrition in program. Now we can obtain an universal coinductive rule which is provided abstractly into fibrational settings for a singlesorted ICDT.

On the basis of categoretical theory, the corecursive computing of a coinductive data types rises in final coalgebras semantic [2]. Any $\forall \alpha: C \rightarrow I \in \boldsymbol{O} b j \mathbb{B} / I$, for $v F \in \boldsymbol{O} b j \mathbb{B} / I$, we use $F$ to make the corecursive manipulation unfold : $(\alpha \rightarrow F(\alpha)) \rightarrow \alpha \rightarrow v F$ to single-sorted ICDT into the base categories $\mathbb{B} / I$. Any an $F$-coalgebras $(\alpha, r: \alpha \rightarrow F(\alpha))$, unfold $r$ is mapping $r$ to a sole $F$ coalgebras morphism unfold $r: \alpha \rightarrow v F$, which is from $(\alpha$, $r$ ) to the final $F$-coalgebra ( $v F$, out) (Fig. 9). Co-recursive manipulation unfold origining in final coalgebras semantic is a corecursive parameterized manipulation of the ICDT in nature. The corecursive computings have many welldefined properties such as exact semantical description, adaptable expansibility and brief expressing.

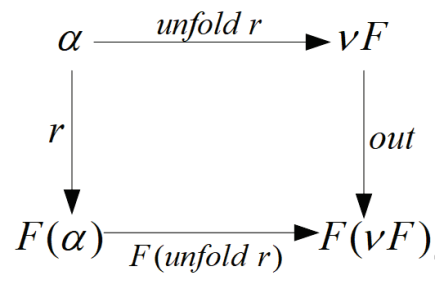

Figure $9 F$-coalgebra morphisms

In Definition 16, we have $E q_{P / I}(F(\alpha)) \cong F^{\perp}\left(E q_{P / I}(\alpha)\right)$, $E q_{P / I}(F(v F)) \cong F^{\perp}\left(E q_{P / I}(v F)\right)$, and the equation functor $E q_{P / I}$, preserves terminal objects. Clearly $E q_{P / I}(v F)$ is one carrier of final $F^{\perp}$-coalgebras, writting it for $v F^{\perp}=E q_{P / I}(v F)$, let $X=E q_{P / I}(\alpha)$. Using selffunctor $F^{\perp}$ makes the corecursive manipulation unfold : $\left(X \rightarrow F^{\perp}(X)\right) \rightarrow X \rightarrow v F^{\perp}$ for a single-sorted ICDT in the total categories $\left.\operatorname{Rel}\left(\mathbb{T} / T_{P}(I)\right)\right)$ (Fig. 10).

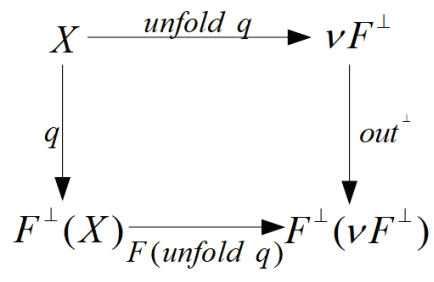

Figure $10 F^{\perp}$-coalgebra morphisms

An $F^{\perp}$ - coalgebras $\left(X, q: X \rightarrow F^{\perp}(X)\right)$, and unfold $q$ is mapping $q$ to a sole $F^{\perp}$ - coalgebras morphism unfold $q$ $X \rightarrow v F^{\perp}$ from $F^{\perp}$ - coalgebras $(X, q)$ to the final $F^{\perp}$ - coalgebras $\left(v F^{\perp}, o u t^{\perp}\right)$. For $\forall \alpha \in \boldsymbol{O} b j \mathbb{B} / I, \quad \exists X \in \boldsymbol{O} b j$ $\operatorname{Rel}\left(\mathbb{T} / T_{P}(I)\right)$, we can obtain an universal coinductive rule for a single-sorted ICDT.

$$
\text { Coind }_{U n i}:\left(X \rightarrow F^{\perp}(X)\right) \rightarrow X \rightarrow E q_{P / I}(v F) .
$$

If $\left(X, q: X \rightarrow F^{\perp}(X)\right)$ is a $F^{\perp}$ - coalgebra over $F$ coalgebras $(\alpha, r: \alpha \rightarrow F(\alpha))$, so Coind $_{U n i} X q$ is a $F^{\perp}$ coalgebras homo-morphism on unfold $r$.

\subsection{Instance Analysis of Single-Sorted ICDT}

Example 4. The type of element of a stream or an infinite sequence is designated by index $I$, such as the natural number Nat, integer Int and character Char, $\forall I \in \boldsymbol{O} b j \mathbb{B}$. For any stream $\alpha: S \rightarrow I \in \boldsymbol{O} b j \mathbb{B} / I$, selffunctor $F: \alpha \rightarrow I \times \alpha$ over $\mathbb{B} / I$, the operation head: $\alpha \rightarrow I$ is head function, another operation tail: $\alpha \rightarrow \alpha$ is tail function after erasing the first item. Any streams properties $R \in \boldsymbol{O b j} \operatorname{Rel}\left(\mathbb{T} / T_{P}(I)\right)$ into the total categories $\operatorname{Rel}\left(\mathbb{T} / T_{P}(I)\right)$ over the relation fibration $\operatorname{Rel}(P / I)$ for the single-sorted index fibration $P / I$, for instance, bisimulation. For the other stream object $\beta: S^{\prime} \rightarrow I$ into $\mathbb{B} / I$, the coinduction for $\alpha$ and $\beta$ about bisimulation property $R$ is as follows: $R$ will be a relationship of bisimulation among two different streams, i.e., $\alpha$ and $\beta$, if and only if $\forall(\alpha, \beta) \in R$, for $(\operatorname{tail}(\alpha), \operatorname{tail}(\beta)) \in R$, there exsits head $(\alpha)=$ head $(\beta)$.

If stream data $\operatorname{Stream}(I)$ is the carrier $v F$ of final $F$ coalgebras $(v F$, out: $v F \rightarrow F(v F))$ into the base categories $\mathbb{B} / I$, then for every $F$ - coalgebras $(\alpha, r: \alpha \rightarrow F(\alpha))$, then it will be lifted to be an $F^{\perp}$ - coalgebras $\left(X, q: X \rightarrow F^{\perp}(X)\right)$ using relation fibration $\operatorname{Rel}(P / I)$, that is satisfying commutive diagrams, namely, $F \cdot \operatorname{Rel}(P / I)(X)=\operatorname{Rel}(P / I) \cdot F^{\perp}(X)$. The terminal properties of final $F$ - coalgebras define a corecursive manipulation unfold $r$ about Stream $(I)$ that implements the determinism for the single-sorted ICDTs $\operatorname{Stream}(I)$. The other corecursive manipulation for terminality of final $F^{\perp}$ coalgebras depicts the semantics behaviors for Stream(I). If $q$ lies above $r$, then $\operatorname{Coind}_{U n i} X q$ is a $F^{\perp}$ - coalgebra homomorphism on unfold $r$, and traversing every property $R$ into the total categories $\operatorname{Rel}\left(\mathbb{T} / T_{P}(I)\right)$ over corresponding relation fibration $\operatorname{Rel}(P / I)$, for $R \in \operatorname{Obj} \operatorname{Rel}\left(\mathbb{T} / T_{P}(I)\right)$, we can then have a semantics set $\left\{R(X, X) \mid X=E q_{P / I}(\alpha), \forall \alpha \in \boldsymbol{O b j} \mathbb{B} / I\right\} \quad$,that represents behavior of $\operatorname{Stream}(I)$.

Taking Example 4 for an instance, unfold $r$ represents the map relation among stream $\alpha$ and its semantics behaviors vividly. The availablity of unfold $r$ supplies an intuitive way to homo-morphism from coalgebras to final coalgebras, so we can establish the coinduction definition principle. To define function unfold $r: \alpha \rightarrow \operatorname{Stream}(I)$, we only need to construct the corresponding operation $r$ on $\alpha$, and let $(\alpha, r)$ be an $F$ - coalgebra with $F(\alpha)=I \times \alpha$. Meanwhile, we can prove two homomorphisms are equivalent given the uniqueness of unfold $r$. So we have coinduction proof principle to demonstrate $m, n: \alpha \rightarrow \operatorname{Stream}(I)$ is equivalent to each other, we just 
demonstrate $m$ and $n$ are homo-morphism from the identical coalgebras $(\alpha, r)$ to the final $F$ - coalgebras $(\operatorname{Stream}(I)$, out: $\operatorname{Stream}(I) \rightarrow F(\operatorname{Stream}(I)))$ as well, $m$ and $n$ are also the same to unfold $r$.

Example 4 presents some fibrational tools, including single-sorted indexed fibration, equation and quotient functor to analyze semantic behaviors deeply and coinductive rule of stream using fibrations, which establishes a mathematical foundation for researching semantic computing and the logic of program languages.

\section{SEMANTIC BEHAVIORS AND COINDUCTIVE RULE OF MANY-SORTED ICDT}

Modeling based on the slice category $\mathbb{B} / I$ analyzes semantic behaviors and describes the coinductive rule of single-sorted ICDT indexed by $I$. But $I$ is only aimed at a single-sorted ICDTs, so hardly to process a more complicated many-sorted ICDTs effectively including reciprocal recursion types. On the basis of work ahead, we have extended the discrete indexed object $I$ to an indexed category $\mathbb{C}$, constructed a many-sorted indexed fibration, described a many-sorted ICDT in $\mathbb{B}$ indexed by $\boldsymbol{O} b j \mathbb{C}$, made a semantic behavior model of the many-sorted ICDTs in the indexed category $\mathbb{C}$ based on fibration $G: \mathbb{B} \rightarrow \mathbb{C}$ and chose different program logics for different indexes.

\subsection{Fibered Fibration}

Definition 17. Let $P: \mathbb{T} \rightarrow \mathbb{B}$ and $P^{\prime}: \mathbb{T}^{\prime} \rightarrow \mathbb{B}$ be two fibrations between small categories. Also, let a fibered functor $F: \mathbb{T} \rightarrow \mathbb{T}^{\prime}$ from $P$ to $P^{\prime}$ above the base category $\mathbb{B}$ satisfy diagram commuting, $P=P^{\prime} \cdot F$. Then $F$ preserves the Cartesian arrow.

Definition 18. Let $F: \mathbb{T} \rightarrow \mathbb{T}^{\prime}$ and $G: \mathbb{T}^{\prime} \rightarrow \mathbb{T}$ be two fibered functors above the base category $\mathbb{B}$. The fibred functor $G$ is a right fibered adjoint functor to $F$, and $F \dashv G$ is a pair of fibred adjunction above $\mathbb{B}$, if $G$ is a right adjoint functor to $F$, and the unit or counit of $F \dashv G$ is vertical.

Definition 17 and Definition 18 lift standard category structures to fibered structures; it is easy to process many practical problems of many-sorted ICDTs with different discrete indexed objects in computer science. Using fibrational tools, such as truth and quotient functors in the base category, we can combine many-sorted ICDTs with their semantic behaviors. Applying reindexed and opposite reindexed functors between fibers in the total category in order to analyze the deeply semantic behaviors of manysorted ICDTs, in order to construct corecursive operations on many-sorted ICDTs to abstractly describe coinductive rules with universality. This does not depend on particular computing environments, but improves the cohesion of many-sorted ICDTs, and further enhances the independence of program languages.

Let $P: \mathbb{T} \rightarrow \mathbb{B}$ and $G: \mathbb{B} \rightarrow \mathbb{C}$ be two fibrations between small categories. Given the composed property that composition of two fibrations is a fibration [23], GP is a fibration. For $\forall a \in \boldsymbol{O} b j \mathbb{C}, \mathbb{T}_{a}$ is a fiber in the total category $\mathbb{T}$ on fibration $G P$ over $a$. The restriction $P_{a}: \mathbb{T}_{a} \rightarrow \mathbb{B}_{a}$ of $P$ at $a$ is a pullback of $P$ along the including functor
Inc: $\mathbb{B}_{a} \rightarrow \mathbb{B}$, and $\mathbb{B}_{a}$ is a fiber in the total category $\mathbb{B}$ on fibration $G$ over $a$. Then given the structure-preserving property of pullbacks [23], $P_{a}$ is also a fibration.

Each $P_{a}$ deals with different indexed object $a$. Let $P$ have a truth functor, so $P_{a}$ also has a truth functor, denoted as $T_{a}$. For a bifibration $P$ that satisfies the Beck-Chevalley condition in Theorem 2, the right adjoint of the reindexed functor preserves terminal objects. When $a$ iterates each indexed object in the indexed category $\mathbb{C}$, a set of $T_{a}$ constructs the truth functor $T_{P}$, that is, $T_{P}=\left\{T_{a} \mid \forall a \in \boldsymbol{O}\right.$ bj $\left.\mathbb{C}\right\}$.

Differing from $T_{P}$ constructed by $T_{a}$, each restriction $P_{a}$ of $P$ has the truth and comprehension functor, we do not determine $P$ itself has a truth and comprehension functor; otherwise, $P$ has truth and comprehension functor, we also do n'ot determine its restriction $P_{a}$ has a truth and comprehension functor. In the following, we introduce the definition of a fibered fibration and demonstrate the decidability of $P$ and its restriction $P_{a}$ about the existences of the truth and comprehension functor.

Definition 19. Let $P: \mathbb{T} \rightarrow \mathbb{B}$ and $G: \mathbb{B} \rightarrow \mathbb{C}$ be two fibrations between small categories, let $T_{P}: \mathbb{B} \rightarrow \mathbb{T}$ be a truth functor for $P$, let $T_{P}$ have one fibered right adjoint functors $\{-\}: G P \rightarrow G$, and $\{-\}$ preserves the Cartesian arrow, then $P$ is a fibered fibration with a truth functor $T_{P}$ and a comprehension functor $\{-\}$ over $G$.

Given Definition 18 and Definition 17, the truth functor $T_{P}: G \rightarrow G P$ of $P$ is a fibered fibration, $T_{P}$ is fibered right adjoint of $P, P$ preserves the opposite Cartesian arrow and $T_{P}$ preserves the Cartesian arrow. Consequently, it is equivalent that $P$ is a fibered fibration over $G$ and that $P$ is a fibration with a truth and comprehension functor. Then according to Theorem 4 below, we delve deeper into the decidability of fibered fibration $P$ and its restriction $P_{a}$ at $a$.

Theorem 4. Let $P: \mathbb{T} \rightarrow \mathbb{B}$ and $G: \mathbb{B} \rightarrow \mathbb{C}$ be two fibrations between small categories, let $P$ be a fibered fibration over $G$. Then for $\forall a \in \boldsymbol{O} b j \mathbb{C}$, a restriction $P_{a}: \mathbb{T}_{a} \rightarrow \mathbb{B}_{a}$ of $P$ at $a$ is also a fibered fibration.

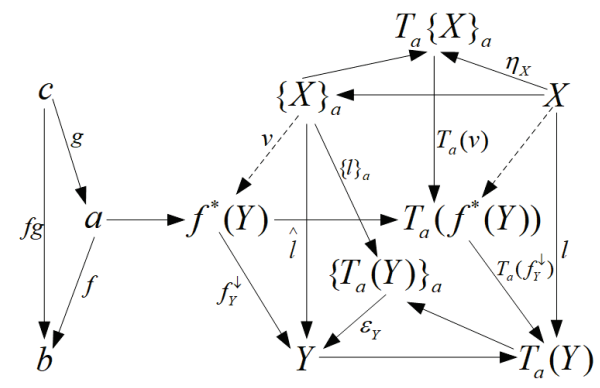

Figure 11 Truth functor $T_{a}$ preserves Cartesian arrows

Proof. Let the fibered adjunction $T_{P} \dashv\{-\}$ be the truth and comprehension functor of the fibered fibration $P$, respectively. For $\forall a \in \boldsymbol{O} b j \mathbb{C}, T_{a}$ and $\{-\}_{a}$ is the restriction of $T_{P}$ and $\{-\}$ at $a$, respectively. With regard to any morphism $f: a \rightarrow b \in \operatorname{Mor} \mathbb{C}, f_{Y}^{\downarrow}: f^{*}(Y) \rightarrow Y \in \operatorname{Mor} \mathbb{B}_{a}$ is a Cartesian arrow of $f$ on fibration $G$. Now we prove that $T_{a}\left(f_{Y}^{\downarrow}\right)$ is also a Cartesian arrow of $f$ on fibration $G P$, i.e., 
truth functor $T_{a}$ preserves the Cartesian arrow. $\exists g: c \rightarrow a \in$ Mor $\mathbb{C}, \quad$ let $l: X \rightarrow T_{a}(Y) \in$ Mor $\mathbb{T}_{a}$ lies above $f g$ (Fig. 11).

Let $\eta: \mathbf{1}_{\mathbb{T}_{a}} \rightarrow T_{a}\{-\}_{a}$ and $\varepsilon:\{-\}_{a} T_{a} \rightarrow \mathbf{1}_{\mathbb{B}_{a}}$ be two natural transformations, and let the transpose $\hat{l}=\varepsilon_{Y}\{l\}_{a}$ of $l$ lies above $f g$. Next, in fiber $\mathbb{B}_{a}$ there exists a unique morphism $v:\{X\}_{a} \rightarrow f^{*}(Y) \in$ Mor $\mathbb{B}_{a}$ over $g$ such that $f_{Y}^{\downarrow} \cdot v=\hat{l}$. Henceforth, in fiber $\mathbb{T}_{a}$ we obtain a unique morphism $\left(T_{a}(v)\right) \eta_{X}: X \rightarrow T_{a}\left(f^{*}(Y)\right) \in \boldsymbol{M o r} \mathbb{T}_{a} \quad$ over $\quad g$, such that $T_{a}\left(f_{Y}^{\downarrow}\right) \cdot\left(T_{a}(v) \eta_{X}\right)=l$. So $T_{a}\left(f_{Y}^{\downarrow}\right)$ is a Cartesian arrow of $f$ on the fibration GP. Namely, the truth functor $T_{a}$ preserves the Cartesian arrows. Similarly, we can also prove the comprehension functor $\{-\}_{a}$ preserves the opposite Cartesian arrows by dual principles in [26].

Therefore, we proved $T_{a} \dashv\{-\}_{a}, \eta$ and $\varepsilon$ are the unit and counit of this adjunction, and $\eta$ is the vertical morphism; the restriction $P_{a}: \mathbb{T}_{a} \rightarrow \mathbb{B}_{a}$ of $P$ at $a$ is also a fibered fibration.

Remark 4. Fibration $G: \mathbb{B} \rightarrow \mathbb{C}$ depicts the indexed type, and Theorem 4 ensures if $P: \mathbb{T} \rightarrow \mathbb{B}$ is a fibered fibration over $G$, then for $\forall a \in \boldsymbol{O} b j \mathbb{C}$, the restriction $P_{a}: \mathbb{T}_{a} \rightarrow \mathbb{B}_{a}$ of $P$ at $a$ is also a fibered fibration with a truth functor $T_{a}$ and a comprehension functor $\{-\}_{a}$, and $T_{a} \dashv$ $\{-\}_{a}$. In fact, $P_{a}$ is a subfibration of $P$ [29], i.e., $P_{a}$ and $P$ have the same fibration structures, semantic behaviors and logical properties. Similarly to subsection 4.1.2 and 4.1.3, the following are some tools of fibration, including: equation functor, quotient functor and lifting equationpreserving of many-sorted indexed fibration $P_{a}$.

\subsection{Semantic Behaviours of Many-Sorted ICDT}

Definition 20. Let $P: \mathbb{T} \rightarrow \mathbb{B}$ be a bifibration satisfying Beck-Chevalley condition, let $P_{a}$ be the restriction of $P$ at $a$. Base category $\mathbb{B}$ has products and pullbacks, $G: \mathbb{B} \rightarrow \mathbb{C}$ is a fibration in the indexed category $\mathbb{C}$, and $P$ is a fibered fibration on $G$ with truth functor $T_{P}$ and comprehension functor $\{-\}$. The diagonal endo-functor $\Delta_{G}: \mathbb{B}_{a} \rightarrow \mathbb{B}_{a}$ maps $\forall C \in \boldsymbol{O} b j \mathbb{B}_{a}$ to $C \times C$, the change of base of $P_{a}$ along $\Delta_{G}$ constructs a relation fibration $\operatorname{Rel}_{G}\left(P_{a}\right): \operatorname{Rel}_{G}\left(\mathbb{T}_{a}\right) \rightarrow \mathbb{B}_{a}$ on $G$. Let $\delta_{G}: I d_{\mathbb{B}_{a}} \rightarrow \Delta_{G}$ be natural transformation, the equation functor $E q_{P a}: \mathbb{B}_{a} \rightarrow \operatorname{Rel}_{G}\left(\mathbb{T}_{a}\right)$ of $P_{a}$ on $G$ maps $C$ to ${ }^{*} \delta_{G} \cdot T_{a}(C)$. If $E q_{P a}$ has a left adjoint $Q_{P a}$, i.e., $Q_{P a} \dashv$ $E q_{P a}$, then $Q_{P a}$ is the quotient functor of $P_{a}$ on $G$.

Definition 21. For $\forall a \in \boldsymbol{O} b j \mathbb{C}$ is an indexed object, the many-sorted indexed fibration $P_{a}: \mathbb{T}_{a} \rightarrow \mathbb{B}_{a}$ is the restriction of $P$ at $a$. Let $F: \mathbb{B}_{a} \rightarrow \mathbb{B}_{a}$ be an endo-functor in fiber $\mathbb{B}_{a}, F_{G}^{\perp}: \operatorname{Rel}_{G}\left(\mathbb{T}_{a}\right) \rightarrow \operatorname{Rel}_{G}\left(\mathbb{T}_{a}\right)$ be a lifting equationpreserving of $F$ on $\operatorname{Rel}_{G}\left(P_{a}\right)$. If it satisfies diagram commuting, i.e., $\operatorname{Rel}_{G}\left(P_{a}\right) \cdot F_{G}^{\perp}=F \cdot \operatorname{Rel}_{G}\left(P_{a}\right)$, then $E q_{P a} \cdot F \cong F_{G}^{\perp} \cdot E q_{P a}$ and $Q_{P a} \cdot F_{G}^{\perp} \cong F \cdot Q_{P a}$.

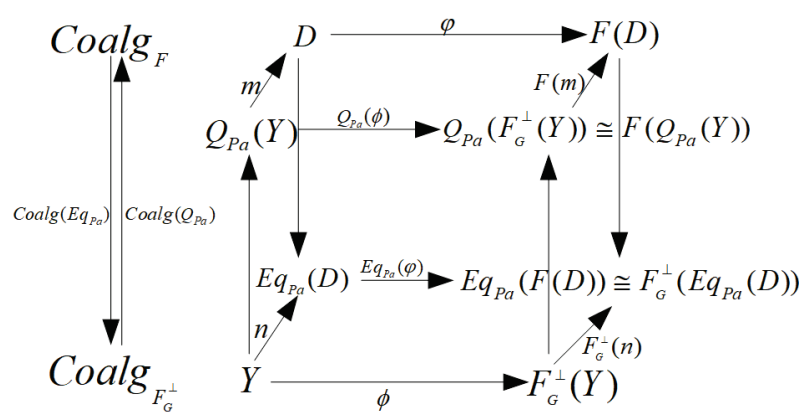

Figure 12 Adjoint properties of $\operatorname{Coalg}\left(E q_{P a}\right)$ and $\operatorname{Coalg}\left(Q_{P a}\right)$

For $\forall D \in \boldsymbol{O} b j \mathbb{B}_{a}$, we can construct an $F$ - coalgebra $(D, \varphi: D \rightarrow F(D))$ through the action of endo-functor $F$. The equation functor $E q_{P a}$ of the many-sorted indexed fibration $P_{a} \quad$ maps $\quad(D, \varphi)$ to $\quad$ a $\quad F_{G}^{\perp} \quad$ - coalgebra $\left(E q_{P a}(D), E q_{P a}(D) \rightarrow F_{G}^{\perp}\left(E q_{P a}(D)\right)\right)$. If $v F_{a}$ have a carrier of final $F$ - coalgebras $\left(v F_{a}\right.$, out $\left.t_{a}: v F_{a} \rightarrow F\left(v F_{a}\right)\right)$. Then the action of $v F_{a}$ by $E q_{P a}$, namely, $E q_{P a}\left(v F_{a}\right)$ is the carrier of terminal $F_{G}^{\perp}-$ coalgebra $\left(v F_{G}^{\perp}, o u t_{G}^{\perp}: v F_{G}^{\perp} \rightarrow F_{G}^{\perp}\left(v F_{G}^{\perp}\right)\right)$ since the equation functor $E q_{P a}$ preserves terminal objects. Similarly for subsection 4.1.4, we write $\operatorname{Coalg}\left(E q_{P a}\right)$ for the functor from $\operatorname{Coalg}_{F}$ to $\operatorname{Coalg}_{F_{\bar{G}}^{\perp}}, \operatorname{Coalg}\left(E q_{P a}\right)$ $\left(\right.$ out $\left._{a}\right)=$ out $_{a}^{\perp} ;$ out $_{a}^{\perp}$ is the isomorphism mapping to out ${ }_{a}$ with the acting on the functor $\operatorname{Coalg}\left(E q_{P a}\right)$.

For any $F_{G}^{\perp}$ - coalgebra $\left(Y, \phi: Y \rightarrow F_{G}^{\perp}(Y)\right)$, the quotient functor $Q_{P a}$ of the many-sorted indexed fibration $P_{a}$ maps $(Y, \phi) \quad$ to $\quad$ a $\quad F \quad$ - $\quad$ coalgebra $\left(Q_{P a}(Y), Q_{P a}(\phi): Q_{P a}(Y) \rightarrow F\left(Q_{P a}(Y)\right)\right) \quad$ (Fig. 12). Let $n: Y \rightarrow E q_{P a}(D)$ be an $F_{G}^{\perp}$ - coalgebras morphism from $\phi$ to $E q_{P a}(\varphi)$, so its corresponding $F$ - coalgebras morphism $m: Q_{P a}(Y) \rightarrow D$ from $Q_{P a}(\varphi)$, to $\varphi$ is a $F$ - coalgebra homomorphism over $n$. Similarly, $n$ is a $F_{G}^{\perp}$ - coalgebra homomorphism over $m$. Functor $\operatorname{Coalg}\left(Q_{P a}\right)$. from Coalg $_{F_{G}^{\perp}}$ to Coalg $_{F}$ establishes an intuitive mutual derivation relationship between $F_{G}^{\perp}$ - coalgebra, whose carrier is $Y$ and $F$ - coalgebra whose carrier is $Q_{P a}(Y)$. This presents a succinct and coherent model for describing coinductive rule of many-sorted ICDTs, with $v F_{a}$ as the carrier of terminal $F$ - coalgebra. If the functor $\operatorname{Coalg}\left(E q_{P a}\right)$ preserves terminal objects, then the lifting equationpreserving $F_{G}^{\perp}$ of $F$ on $\operatorname{Rel}_{G}\left(P_{a}\right)$ generates a sound coinductive rule.

\subsection{Coinductive Rule of Many-Sorted ICDT}

Let $P: \mathbb{T} \rightarrow \mathbb{B}$ and $G: \mathbb{B} \rightarrow \mathbb{C}$ satisfy the requirements of Definition 20 and Definition 21; let $F: \mathbb{B}_{a} \rightarrow \mathbb{B}_{a}$ be an endo-functor in fiber $\mathbb{B}_{a}$, and $v F_{a}$ is a carrier of final $F$ coalgebras. Each lifting which is equation-preserving $F_{G}^{\perp}: \mathbb{T}_{a} \rightarrow \mathbb{T}_{a}$ of $F$ has a sound coinductive rule about $v F_{a}$, so it ensures the validity of the coinductive rule generated by the many-sorted indexed fibration $P_{a}$ on a many-sorted 
ICDT. Next we will present and describe the coinductive rule of many-sorted ICDTs with universality in the settings of fibrations.

For $\forall a \in \boldsymbol{O} b j \mathbb{C}, \forall D \in \boldsymbol{O} b j \mathbb{B}_{a},(D, \varphi: D \rightarrow F(D))$ is a $F$ coalgebra in fiber $\mathbb{B}_{a}$. We construct a corecursive operation unfold: $(D \rightarrow F(D)) \rightarrow D \rightarrow v F_{a}$ of a many-sorted ICDT in the base category $\mathbb{B}_{a}$ on relation fibration $\operatorname{Rel}_{G}\left(P_{a}\right)$, and unfold $\varphi$ maps $\varphi$ to a sole $F$ - coalgebras morphism unfold $\varphi: D \rightarrow v F_{a}$ from $(D, \varphi)$ to its final $F-$ coalgebras $\left(v F_{a}, o u t_{a}\right)$.

Given property that the equation functor $E q_{P a}$ preserves terminal objects, and $E q_{P a}\left(v F_{a}\right)$ is the carrier of terminal $F_{G}^{\perp}$ - coalgebra, write $v F_{a}^{\perp}=E q_{P a}\left(v F_{a}\right)$. The isomorphism expression is as the following: $E q_{P a}\left(F\left(v F_{a}\right)\right) \cong F_{G}^{\perp}\left(E q_{P a}\left(v F_{a}\right)\right)=F_{G}^{\perp}\left(v F_{a}^{\perp}\right)$. A corecursive operation of a many-sorted ICDT unfold: $\left(Y \rightarrow F_{G}^{\perp}(Y)\right) \rightarrow Y \rightarrow v F_{a}^{\perp}$ is constructed by $F_{G}^{\perp}$ in the total category $\operatorname{Rel}_{G}\left(\mathbb{T}_{a}\right)$ on relation fibration $\operatorname{Rel}_{G}\left(P_{a}\right)$. For any $F_{G}^{\perp}$ - coalgebra $\left(Y, \phi: Y \rightarrow F_{G}^{\perp}(Y)\right)$, unfold $\phi$ maps $\phi$ to a unique $F_{G}^{\perp}$ - coalgebra morphism unfold $\phi: Y \rightarrow v F_{a}^{\perp}$ from $(Y, \phi)$ to terminal $F_{G}^{\perp}$ - coalgebra $\left(v F_{a}^{\perp}, o u t_{a}^{\perp}: v F_{a}^{\perp} \rightarrow F_{G}^{\perp}\left(v F_{a}^{\perp}\right)\right)$. For $\forall D \in \boldsymbol{O} b j \mathbb{B}_{a}, \forall a \in \boldsymbol{O} b j \mathbb{C}$, $\exists Y \in \operatorname{Obj~Rel}_{G}\left(\mathbb{T}_{a}\right)$, a coinductive rule of many-sorted ICDT with universality is as follows:

$$
\text { Coind }_{U n i}^{\prime}:\left(Y \rightarrow F_{G}^{\perp}(Y)\right) \rightarrow Y \rightarrow E q_{P a}\left(v F_{a}\right) \text {. }
$$

If $\phi: Y \rightarrow F_{G}^{\perp}(Y)$ is an $F_{G}^{\perp}$ - coalgebra over the $F$ coalgebra $(D, \varphi: D \rightarrow F(D))$, then Coind $_{U n i} Y \phi$ is a $F_{G}^{\perp}$ coalgebra homomorphism over unfold $\varphi$.

\subsection{Instance Analysis of Many-Sorted ICDT}

Example 5. For any set $A$, let $L^{\infty}=L^{N} \cup L^{\omega}$ be the partial order set, including all elements of $A$, where $L^{N}$ is an infinite set and $L^{\omega}$ is a finite set. In turn, we have taken corresponding elements from the even and odd position of $L^{\infty}$ to form two partial order sets $E V E N$ and $O D D$, with two functions even: $L^{\infty} \rightarrow$ EVEN and odd: $L^{\infty} \rightarrow O D D$. Then EVEN and $O D D$ are mutual recursive many-sorted ICDTs. Let $a$, $b$ be two indexed objects only in the indexed category $\mathbb{C}$, $a$ is the indexed object of $E V E N$, and $b$ is the indexed object of $O D D$. We have defined the endo-functor $F: \mathbb{B} \times \mathbb{B} \rightarrow \mathbb{B} \times \mathbb{B}$ in the base category $\mathbb{B} \times \mathbb{B}$, which is a binary production. For $\forall E \in E V E N, \forall O \in O D D$, we have $F(E, O)=(O, E)$. Write - for the merging operation of elements; we have defined the merging property merge: $\operatorname{merge}(x \cdot E V E N, O D D)=x \cdot \operatorname{merge}(O D D, E V E N)$

$\operatorname{even}(\operatorname{merge}(E V E N, O D D))=E V E N$

and

$\operatorname{odd}(\operatorname{merge}(E V E N, O D D))=O D D$. Therefore, the relation on $\begin{array}{lllll}\text { carrier } & L^{\infty} & \text { of } & F & -\end{array}$ coalgebra $R=\{(E V E N, \operatorname{even}(\operatorname{merge}(E V E N, O D D)))\}$ and

$S=\{(O D D o d d(\operatorname{merge}(E V E N, O D D)))\}$ are a bisimulation.

Let $(E V E N, O D D)$ be the carrier $\left(v F_{E}, v F_{O}\right)$ of terminal $F$ - coalgebra over binary productions in the base category on relation fibration $\left(\operatorname{Rel}_{G}\left(P_{a}\right), \operatorname{Rel}_{G}\left(P_{b}\right)\right)$ of the manysorted indexed fibration $\left(P_{a}, P_{b}\right)$. For any $F$ - coalgebra $((E, O), m:(E, O) \rightarrow F(E, O))$, is lifted to be a $F_{G}^{\perp}$ - coalgebra $\left(\left(Y, Y^{\prime}\right), \phi:\left(Y, Y^{\prime}\right) \rightarrow F_{G}^{\perp}\left(Y, Y^{\prime}\right)\right) \quad$ by $\left(\operatorname{Rel}_{G}\left(P_{a}\right), \operatorname{Rel}_{G}\left(P_{b}\right)\right)$. This satisfies diagram commuting $\left(F \cdot\left(\operatorname{Rel}_{G}\left(P_{a}\right), \operatorname{Rel}_{G}\left(P_{b}\right)\right)\right)(R, S)=\left(\left(\operatorname{Rel}_{G}\left(P_{a}\right), \operatorname{Rel}_{G}\left(P_{b}\right)\right) \cdot F_{G}^{\perp}\right)(R, S)$

- A corecursive operation unfold $\varphi$ is defined by the terminality of terminal $F$ - coalgebra on (EVEN, ODD), executing the judgment of a many-sorted ICDT(EVEN, $O D D)$; another corecursive operation defined by the terminality of terminal $F_{G}^{\perp}$ - coalgebra depicts semantic behaviors of $(E V E N, O D D)$. If $\phi$ lies above $\varphi$, then Coind ${ }_{U n i}(R, S) \phi$ is an $F_{G}^{\perp}$ - coalgebra homomorphism over unfold $\varphi$. When iterating each property $R \in \operatorname{Obj}_{\operatorname{Rel}_{G}}\left(\mathbb{T}_{a}\right), S \in \operatorname{Obj}_{\operatorname{Rel}_{G}}\left(\mathbb{T}_{b}\right), \forall a, b \in \boldsymbol{O b j} \mathbb{C}$ in the total category $\left(\operatorname{Rel}_{G}\left(\mathbb{T}_{a}\right), \operatorname{Rel}_{G}\left(\mathbb{T}_{b}\right)\right)$ on relation fibration $\left(\operatorname{Rel}_{G}\left(P_{a}\right), \operatorname{Rel}_{G}\left(P_{b}\right)\right)$, we obtain the semantic set describing properties of $(E V E N, \quad O D D)$, that is, $\left\{\left(R(Y), S\left(Y^{\prime}\right)\right) \mid Y=E q_{P a}(E), Y^{\prime}=E q_{P b}(O)\right\}$.

The mutual recursive type is a complex many-sorted ICDT. Traditional methods, including algebras and category theory, are difficult when effectively processing their semantic computing and program logic. Example 5 analyzes the deeply semantic properties of mutual recursive type using fibrations. The fibrational method is not strictly dependent on particular methods or tools, such as predicate logic or set theory, and abstractly depicts its coinductive rule with universality. Example 5 expands and deepens traditional methods in the level of category theory. It deals with the semantic computation of the mutual recursive type in the uniform settings of fibrations, and further develops the width and depth of traditional methods of ICDT in math.

\section{CONCLUSIONS}

Fibrations integrate conventional ideology regarding programming, with special ideas and studying methods, such as highly abstract, nimble development and brief description, produces a robust and significant effect on program languages and formal semantics, and boosts the application of categoretical theory in computer science. There is little literature on fibrations in computer science, especially regarding systematical and deep research aiming at programming; there is even less literature relating to its formal semantics. Fibrations have special superiorities in resolving the represention of speculative matters. At the same time, they are important in the application of theoretical computer science. This paper executed some preliminary works in analyzing semantical behaviors, coinductive rules representation of ICDTs. In general, we expect this work can promote interest for academics particularly in China regarding fibrational method, promoting the prospects of fibration itself and their applications in computer science.

Our future work will be a preliminary discussion on the soundness, completeness, and consistency of a formal system consisting of ICDT and its coinductive rule. Furthermore, we are expanding our ICDT work to include 
2-categories using fibrations, with deep discussion regarding math structures and the categorical properties of syntax construction, semantics computation, behavoirs description and programming logic in 2-categories.

\section{Acknowledgements}

Our works are supported by the GuangDong Provincial Natural Science Foundations, China PRC (No: 2018 A0303130274), GuangDong Provincial Institutions of Higher Learning Prominent Youth Teachers Train Project, China PRC (No: YQ2014155).

\section{REFERENCES}

[1] Greiner, J. (1992). Programming with inductive and coinductive types. Pittsburgh, USA: Carnegie Mellon University. https://doi.org/10.21236/ADA249562

[2] Rutten, J. J. M. M. (2000). Universal coalgebra: a theory of systems.Theoretical Computer Science, 249(1), 3-80. https://doi.org/10.1016/s0304-3975(00)00056-6

[3] Zhou, X. C. \& Shu, Z. M. (2003). A survey on the coalgebraic methods in computer science. Journal of Software, 14(10), 1661-1671.

[4] Johnson, M. \& Rosebrugh, R. (2007). Fibrations and universal view updatability. Theoretical Computer Science, 388(1-3), 109-129. https://doi.org/10.1016/j.tcs.2007.06.004

[5] Johnson, M., Rosebrugh, R., \& Wood, R. J. (2012). Lenses, fibrations and universal translations.Mathematics Structure in Computer Science, 22(1), 25-42. https://doi.org/10.1017/s0960129511000442

[6] Tews, H. (2002). Coalgebra method for object-oriented specification. Dresden, Germany: Institute Theoretische Informatik, Technischen Universiy.

[7] Ghani, N., Johann P., \& Fumex, C. (2012). Generic fibrational induction. Logical Methods in Computer Science, 8(2), 1-27. https://doi.org/10.2168//mcs-8(2:12)2012

[8] Miao, D. C., Xi, J. Q., Jia, L. Y., \& Liu, Y. (2011). Formal language algebraic model. Journal of South China University of Technology (Natural Science Edition), 39(10), 74-78.

[9] Hermida, C. \& Jacobs, B. (1998).Structural induction and coinduction in a fibrational setting. Information and Computation, 145(2), 107-152. https://doi.org/10.1006/inco.1998.2725

[10] Hagino, T. (1987). A categorical programming language. Edinburgh, UK: University of Edinburgh. https://doi.org/10.1016/B978-0-12-037104-4.50012-8

[11] Nogueira, P. \& Moreno-Navarro, J. (2008). Bialgebra views: a way for polytypic programming to cohabit with data abstract. Proceedings of the ACM SIGPLAN Workshop on Generic Programming. Victoria, BC: Canada, 61-73. https://doi.org/10.1145/1411318.1411327

[12] Su, J. D. \& Yu, S. S. (2011). Coinductive data types and their applications in programming languages. Computer Science, 38(11), 114-118. https://doi.org/10.1007/s00466-010-0527-8

[13] Poll, E. (1998). Subtyping and inheritance for categorical datatypes. RIMS Lecture Notes, 1023, 112-125.

[14] Hinze, R. (2010). Reasoning about codata. Lecture Notes in Computer Science, 6299, 42-93. https://doi.org/10.1007/978-3-642-17685-2_3

[15] Miao, D. C. \& Xi, J. Q. (2018). Indexed coinduction in a fibrational setting. Lecture Notes in Computer Science, 11338, 10-16. https://doi.org/10.1007/978-3-030-05234-8_2

[16] Gimenez, E. \& Casteran, P. (1998). A turorial on coinductive types in Coq. Retrieved from http:www.labri.fr/perso/ casteran/RecTutorial.pdf, 1998
[17] Vene V. (2000). Categorical programming with inductive and coinductive types. Tartu, Estonia: University of Tartu.

[18] Ghani, N., Revell, T., \& Atkey, R. (2015). Fibrational units of measure. Retrieved from https://personal.cis.strath.ac.uk/neil.ghani/pub.html

[19] Bonchi, F., Petrisan, D., \& Pous, D. (2014). Coinduction upto in a fibrational setting. Proceedings of the Joint Meeting of the $23^{\text {rd }}$ EACSL Annual Conference on Computer Science Logic and the 29th Annual ACM/IEEE Symposium on Logic in Computer Science. New York, 1-18. https://doi.org/10.1145/2603088.2603149

[20] Chen, L.T. \& Urbat, H. (2015). A fibrational approach to automata theory. The 6th International Conference on Algebra and Coalgebra in Computer Science. Dagstuhl, Germany, 50-65.

[21] Worrell, J. (2005). On the final sequence of a finitary set functor. Theoretical Computer Science, 338(1-3), 184-199. https://doi.org/10.1016/.tcs.2004.12.009

[22] Hasuo, I., Kataoka, T., \& Cho, K. (2018). Coinductive predicates and final sequences in a fibration. Mathematics Structure in Computer Science, 28(4), 562-611. https://doi.org/10.1017/s0960129517000056

[23] Barr, M. \& Wells, C. (1990). Category Theory for CompuerScience. NewYork: Prentice-Hall Books.

[24] Pavlovic, D. (1990). Predicates and fibrations. Utrecht, Nederland: University of Utrecht.

[25] Jacobs, B. (2001). Categorical Logic and Type Theory. Amsterdam, Nederland: Elsevier Science Books.

[26] He, W. (2006). Category Theory. Beijing: Science Press Books.

[27] Morris, P. \& Altenkirch, T. (2009). Indexed containers. Proceedings of the $24^{\text {th }}$ Annual IEEE Symposium on Logic in Computer Science. Los Angeles: CA, 277-285. https://doi.org/10.1109/lics.2009.33

[28] Ghani, N., Johann, P., \& Fumex, C. (2013). Indexed induction and coinduction, fibrationally. Logical Methods in Computer Science, 9(3-6), 1-31. https://doi.org/10.2168//mcs-9(3:6)2013

[29] Hermida, C. (1993). Fibrations, Logical predicates and indeterminates. Edinburgh, UK: University of Edinburgh. https://doi.org/10.7146/dpb.v22i462.6935

\section{Contact information}

Decheng MIAO, professor

(Corresponding author)

School of Information Science and Engineering, Shaoguan University No. 288, Daxue Road, Zhenjiang District, Shaoguan, China

E-mail: tony10860@126.com

Chaoyang WANG, master candidate

School of Journalism and Communication, Guangzhou University, No. 230, Waihuanxi Road, Panyu District,Guangzhou, China

E-mail: wcy604484222@163.com

\section{Xinsheng LIU, lecturer}

Army Artillery and Air Defense Force Academy,

No. 31, Dongdaying Road, Shenhe District, Shenyang, China

E-mail: waddddaw@126.com

Yonglin LIANG, associate professor

School of Information Science and Engineering, Shaoguan University,

No. 288, Daxue Road, Zhenjiang District, Shaoguan, China

E-mail: liangyonglin@126.com 